\title{
Modified wave operators without loss of regularity for some long range Hartree equations. I
}

\author{
J. Ginibre \\ Laboratoire de Physique Théorique* \\ Université de Paris XI, Bâtiment 210, F-91405 ORSAY Cedex, France \\ G. Velo \\ Dipartimento di Fisica, Università di Bologna \\ and INFN, Sezione di Bologna, Italy
}

\begin{abstract}
We reconsider the theory of scattering for some long range Hartree equations with potential $|x|^{-\gamma}$ with $1 / 2<\gamma<1$. More precisely we study the local Cauchy problem with infinite initial time, which is the main step in the construction of the modified wave operators. We solve that problem in the whole subcritical range without loss of regularity between the asymptotic state and the solution, thereby recovering a result of Nakanishi. Our method starts from a different parametrization of the solutions, already used in our previous papers. This reduces the proofs to energy estimates and avoids delicate phase estimates.
\end{abstract}

2000 MSC : Primary 35P25. Secondary 35B40, 35Q40, 81 U99.

Key words : Long range scattering, wave operators, Hartree equation.

LPT Orsay 12-33

April 2012

${ }^{*}$ Unité Mixte de Recherche (CNRS) UMR 8627 


\section{Introduction}

This paper is devoted to the theory of scattering and more precisely to the proof of existence of modified wave operators for the long range Hartree type equation

$$
i \partial_{t} u=-(1 / 2) \Delta u+g(u) u
$$

where $u$ is a complex valued function defined in space time $I R^{n+1}$ with $n \geq 2, \Delta$ is the Laplace operator in $I^{n}$ and

$$
g(u)=\kappa|x|^{-\gamma} \star|u|^{2}
$$

where $\kappa \in \mathbb{R}, 0<\gamma \leq 1$ and $\star$ denotes the convolution in $\mathbb{I R}^{n}$.

A large amount of work has been devoted to the theory of scattering for the Hartree equation (1.1) with nonlinearity (1.2), both in the short range case $\gamma>1$ and in the long range case $\gamma \leq 1$. See [2] [3] [5] [6] and references therein quoted. In order to prove the existence of wave operators, one has to construct solutions of the given equation with prescribed asymptotic behaviour at $\pm \infty$ in time. The asymptotic behaviour is that of solutions of the free Schrödinger equation in the short range case $\gamma>1$, thereby leading to ordinary wave operators, and has to be modified by a suitable phase factor in the long range case $\gamma \leq 1$, thereby leading to modified wave operators in that case. The asymptotic behaviour is parametrized by an asymptotic state, which plays the role of (in the short range case can be taken to be) the initial data for the asymptotic behaviour. The main step in the construction of solutions with prescribed asymptotic behaviour consists in solving the local Cauchy problem with infinite initial time, with the asymptotic state playing the role of initial data. From now on, we concentrate on that problem. In [2] we have solved that problem for arbitrarily large data in the range $1 / 2<\gamma<1$ (the easier borderline case $\gamma=1$ can be treated by the same method but requires slightly different formulas containing logarithms). The method used in [2] consists in parametrizing the solution $u$ in terms of an amplitude $v$ and a phase $\varphi$ and solving an auxiliary system of equations for the pair $(v, \varphi)$. It has two defects, namely : (i) there is a natural notion of criticality for that problem, and the regularity required for the solution is significantly higher than the critical one, namely the method does not cover the entire subcritical range, and (ii) there occurs a loss of regularity (essentially a loss of two derivatives on $v$ ) between the asymptotic state and the solution eventually obtained. These two defects were remedied in [5] and [6] for $\gamma=1$ and for $1 / 2<\gamma<1$ respectively, 
by the use of two new ingredients, namely : (i) a different parametrization of the solution $u$, and (ii) the clever use of a local conservation law satisfied by Schrödinger type equations including (1.1). This allows in addition to fix the new phase $\varphi$ from the beginning, thereby leaving only one equation for the new amplitude $v$. However the method requires rather complicated phase estimates in the more difficult case $1 / 2<\gamma<1$.

It turns out however that the two new ingredients used in [5] [6] are independent of each other. In particular the local conservation law can also be exploited with the parametrization used in [2], provided the latter is supplemented with the systematic use of an ultraviolet momentum cut-off. One can then recover the main results of [6], namely solve the local Cauchy problem at infinity in time in the whole subcritical range and without any regularity loss, by elementary energy methods not requiring any delicate phase estimates. The purpose of the present paper is to present that simpler alternative method.

The simpler method also lends itself naturally to an iteration scheme which can be expected to cover the range $\gamma \leq 1 / 2$, with the $n$-th interation covering the range $1 /(n+2)<\gamma<1 /(n+1)$, still without any regularity loss between the asymptotic state and the solution. However the method does not seem to make it possible to cover the entire subcritical range as soon as $\gamma \leq 1 / 2$, and stronger regularity conditions seem to be required. Furthermore, the necessary estimates, although still elementary, become more and more cumbersome as $n$ increases. In a subsequent paper, as an illustration we shall treat the problem in the range $1 / 3<\gamma<1 / 2$ by means of the first iteration.

We now introduce the relevant parametrization of $u$ needed to study the Cauchy problem at infinite time, restricting our attention to positive time. The unitary group

$$
U(t)=\exp (i(t / 2) \Delta)
$$

which solves the free Schrödinger equation can be written as

$$
U(t)=M(t) D(t) F M(t)
$$

where $M(t)$ is the operator of multiplication by the function

$$
M(t)=\exp \left(i x^{2} / 2 t\right),
$$

$F$ is the Fourier transform and $D(t)$ is the dilation operator

$$
D(t)=(i t)^{-n / 2} D_{0}(t)
$$


where

$$
\left(D_{0}(t) f\right)(x)=f(x / t) .
$$

For any function $w$ of space time, we define

$$
\widetilde{w}(t)=U(-t) w(t)
$$

and we define the pseudoconformal inverse $w_{c}$ of $w$ by

$$
w(t)=M(t) D(t) \overline{w_{c}}(1 / t)
$$

or equivalently

$$
\widetilde{w}(t)=\overline{F \widetilde{w}_{c}(1 / t)}
$$

which shows that the pseudoconformal inversion is involutive.

The Cauchy problem at infinite initial time for $u$ is then equivalent to the Cauchy problem at initial time zero for its pseudoconformal inverse $u_{c}$. The equation (1.1) is replaced by

$$
i \partial_{t} u_{c}=-(1 / 2) \Delta u_{c}+t^{\gamma-2} g\left(u_{c}\right) u_{c} .
$$

We now parametrize $u_{c}$ in terms of an amplitude $v$ and a phase $\varphi$ according to

$$
u_{c}(t)=\exp (-i \varphi(t)) v(t)
$$

so that

$$
\begin{aligned}
u(t) & =M(t) D(t) \exp (i \varphi(1 / t)) \bar{v}(1 / t) \\
& =D(t) \exp (i \varphi(1 / t)) D^{-1}(t) M(t) D(t) \bar{v}(1 / t)
\end{aligned}
$$

or equivalently

$$
u(t)=\exp \left(i\left(D_{0}(t) \varphi(1 / t)\right)\right) v_{c}(t) .
$$

The original equation then becomes the following equation for $v$

$$
i \partial_{t} v=-(1 / 2) \Delta_{s} v+\left(t^{\gamma-2} g(v)-\partial_{t} \varphi\right) v
$$

where $s=\nabla \varphi$ and

$$
\Delta_{s}=(\nabla-i s)^{2}=\Delta-2 i s \cdot \nabla-i(\nabla \cdot s)-|s|^{2} .
$$

We want to choose $\varphi$ so as to cancel the divergence at $t=0$ of the last term in (1.14), but that cancellation is needed only at large distances, namely 
for low momentum. We therefore introduce a momentum cut-off as follows. Let $\chi \in \mathcal{C}^{\infty}\left(\mathbb{R}^{+}, \mathbb{R}^{+}\right), 0 \leq \chi \leq 1, \chi(\ell)=1$ for $\ell \leq 1, \chi(\ell)=0$ for $\ell \geq 2$. We define

$$
\chi_{L}=\chi\left(\omega t^{1 / 2}\right) \quad, \quad \chi_{S}=1-\chi_{L}
$$

with $\omega=(-\Delta)^{1 / 2}$, and correspondingly

$$
g_{L}(v)=\chi_{L} g(v) \quad, \quad g_{S}(v)=\chi_{S} g(v) .
$$

We want to solve (1.14) with $v$ continuous at $t=0$ with $v(0)=v_{0}$. For that purpose, we choose (assuming from now on $\gamma<1$ )

$$
\varphi=-(1-\gamma)^{-1} t^{\gamma-1} g_{L}\left(v_{0}\right)
$$

so that

$$
\partial_{t} \varphi=t^{\gamma-2} g_{L}\left(v_{0}\right)-(1-\gamma)^{-1} t^{\gamma-2} \tilde{\chi}_{L} g\left(v_{0}\right)
$$

with

$$
\tilde{\chi}_{L}=\tilde{\chi}\left(\omega t^{1 / 2}\right), \widetilde{\chi}(\ell)=(1 / 2) \ell \chi^{\prime}(\ell) .
$$

With that choice, the equation (1.14) becomes

$$
i \partial_{t} v=L(v) v
$$

with

$$
L(v)=-(1 / 2) \Delta_{s}+t^{\gamma-2} g_{S}(v)+(1-\gamma)^{-1} t^{\gamma-2} \tilde{\chi}_{L} g\left(v_{0}\right)+t^{\gamma-2}\left(g_{L}(v)-g_{L}\left(v_{0}\right)\right) .
$$

We shall also need the partly linearized equation for $v^{\prime}$

$$
i \partial_{t} v^{\prime}=L(v) v^{\prime}
$$

The method consists in first solving the Cauchy problem with initial time zero for the linearized equation (1.23). One then shows that the map $v \rightarrow v^{\prime}$ thereby defined is a contraction in a suitable space in a sufficiently small time interval. This solves the Cauchy problem with initial time zero for the nonlinear equation (1.21). One then translates the results through the change of variables (1.12) to solve the Cauchy problem with initial time zero for the equation (1.11) or equivalently with infinite initial time for the equation (1.1). The final result can be stated as the 
following proposition, which is adapted to the equation (1.1) in a neighborhood of infinity in time. We need the notation

$$
F H^{\rho}=\left\{u \in \mathcal{S}^{\prime}: F^{-1} u \in H^{\rho}\right\}
$$

Proposition 1.1. Let $1 / 2<\gamma<1$. Let $1-\gamma / 2<\rho<n / 2$.

(1) Let $u_{0} \in F H^{\rho}$ and define

$$
\varphi(t)=-(1-\gamma)^{-1} t^{\gamma-1} g_{L}\left(F u_{0}\right)
$$

Then there exists $T_{\infty}>0$ and there exists a unique solution $u$ of the equation (1.1) such that $v_{c}$ defined by (1.9) (1.12) or equivalently by (1.13) satisfies $\widetilde{v}_{c} \in$ $\mathcal{C}\left(\left[T_{\infty}, \infty\right), F H^{\rho}\right)$ and such that

$$
\widetilde{v}_{c}(t) \rightarrow u_{0} \text { in } F H^{\rho} \text { when } t \rightarrow \infty
$$

Furthermore $\tilde{u} \in \mathcal{C}\left(\left[T_{\infty}, \infty\right), F H^{\rho}\right)$ and $\widetilde{u}$ satisfies the estimate

$$
\left\|\widetilde{u}(t) ; F H^{\rho}\right\| \leq C a_{0}\left(1+a_{0}^{2} t^{1-\gamma}\right)^{1+[\rho]}
$$

for all $t \geq T_{\infty}$, where $[\rho]$ is the integral part of $\rho$ and

$$
a_{0}=\left\|u_{0} ; F H^{\rho}\right\|
$$

(2) Let in addition $\rho>3 / 4$. Then the map $u_{0} \rightarrow \widetilde{v}_{c}$ is continuous from $F H^{\rho}$ to $L^{\infty}\left(\left[T_{\infty}, \infty\right), F H^{\rho}\right)$ and the map $u_{0} \rightarrow \widetilde{u}$ is continuous from $F H^{\rho}$ to $L^{\infty}\left(\left[T_{\infty}, T_{1}\right], F H^{\rho}\right)$ for all $T_{1}, T_{\infty}<T_{1}<\infty$.

Proposition 1.1 follows from Propositions 4.1, 4.2, 5.1 and 5.2 through the change of variables (1.9) or (1.10), which implies in particular that

$$
\left\|\widetilde{w}(t) ; F H^{\rho}\right\|=\left\|\widetilde{w}_{c}(1 / t) ; H^{\rho}\right\|=\left\|w_{c}(1 / t) ; H^{\rho}\right\|
$$

The condition $\rho>1-\gamma / 2$ in Proposition 1.1 is the subcriticality condition mentioned above. For technical reasons, the continuity properties in Part (2) are proved only under the stronger condition $\rho>3 / 4$.

This paper is organized as follows. In Section 2, we introduce some notation and we collect a number of estimates which are used throughout this paper. In Section 3, we study the Cauchy problem for the linearized equation (1.23) with initial time 
$t_{0} \geq 0$. In Section 4, we solve the Cauchy problem with initial time zero for the nonlinear equation (1.21). In Section 5, we prove the continuity of the solutions of (1.21) with respect to the initial data. In Appendix A1, we prove Lemma 2.4 on commutators. In Appendix A2, we sketch the proof of the preliminary Proposition 3.1.

In all this paper (as in [2]) we assume that $1 / 2<\gamma<1$. The easier case $\gamma=1$ can be treated by the same method, but requires slightly different formulas.

\section{Notation and preliminary estimates}

In this section we introduce some notation and we collect a number of estimates which will be used throughout this paper. We denote by $\|\cdot\|_{r}$ the norm in $L^{r} \equiv$ $L^{r}\left(I^{n}\right)$. For any interval $I$ and any Banach space $X$ we denote by $\mathcal{C}(I, X)$ (resp. $\left.\mathcal{C}_{w}(I, X)\right)$ the space of strongly (resp. weakly) continuous functions from $I$ to $X$ and by $L^{\infty}(I, X)$ the space of measurable essentially bounded functions from $I$ to $X$. For real numbers $a$ and $b$ we use the notation $a \vee b=\operatorname{Max}(a, b)$ and $a \wedge b=\operatorname{Min}(a, b)$. We define $(a)_{+}=a \vee 0$ and

$$
\begin{aligned}
{[a]_{+} } & =(a)_{+} & & \text {for } a \neq 0 \\
& =\varepsilon \text { for some } \varepsilon>0 & & \text { for } a=0 .
\end{aligned}
$$

We shall use the Sobolev spaces $\dot{H}_{r}^{\sigma}$ and $H_{r}^{\sigma}$ defined for $-\infty<\sigma<+\infty$, $1 \leq r<\infty$ by

$$
\dot{H}_{r}^{\sigma}=\left\{u:\left\|u ; \dot{H}_{r}^{\sigma}\right\| \equiv\left\|\omega^{\sigma} u\right\|_{r}<\infty\right\}
$$

and

$$
H_{r}^{\sigma}=\left\{u:\left\|u ; H_{r}^{\sigma}\right\| \equiv\left\|<\omega>^{\sigma} u\right\|_{r}<\infty\right\}
$$

where $\omega=(-\Delta)^{1 / 2}$ and $<\cdot>=\left(1+|\cdot|^{2}\right)^{1 / 2}$. The subscript $r$ will be omitted both in $H^{\sigma}$ and in the $L^{r}$ norm if $r=2$ and we shall use the notation

$$
\left\|\omega^{\sigma \pm 0} u\right\|=\left(\left\|\omega^{\sigma+\varepsilon} u\right\|\left\|\omega^{\sigma-\varepsilon} u\right\|\right)^{1 / 2} \quad \text { for some } \varepsilon>0 .
$$

Note also that for $0<\gamma<n[7]$

$$
g(u)=\kappa|x|^{-\gamma} \star|u|^{2}=\kappa C_{\gamma, n} \omega^{\gamma-n}|u|^{2} .
$$


We shall use extensively the following Sobolev inequalities.

Lemma 2.1. Let $1<q, r<\infty, 1<p \leq \infty$ and $0 \leq \sigma<\rho$. If $p=\infty$, assume that $\rho-\sigma>n / r$. Let $\theta$ satisfy $\sigma / \rho \leq \theta \leq 1$ and

$$
n / p-\sigma=(1-\theta) n / q+\theta(n / r-\rho)
$$

Then the following inequality holds

$$
\left\|\omega^{\sigma} u\right\|_{p} \leq C\|u\|_{q}^{1-\theta}\left\|\omega^{\rho} u\right\|_{r}^{\theta}
$$

We shall also use extensively the following Leibnitz estimates.

Lemma 2.2. Let $1<r, r_{1}, r_{3}<\infty$ and

$$
1 / r=1 / r_{1}+1 / r_{2}=1 / r_{3}+1 / r_{4}
$$

Then the following estimates hold for $\sigma \geq 0$ :

$$
\left\|\omega^{\sigma}(u v)\right\|_{r} \leq C\left(\left\|\omega^{\sigma} u\right\|_{r_{1}}\|v\|_{r_{2}}+\left\|\omega^{\sigma} v\right\|_{r_{3}}\|u\|_{r_{4}}\right) .
$$

An easy consequence of Lemmas 2.1 and 2.2 is the inequality

$$
\begin{aligned}
\left\|\omega^{\sigma} f u\right\| & \leq C\left(\|f\|_{\infty}+\left\|\omega^{n / 2} f\right\|\right)\left\|\omega^{\sigma} u\right\| . \\
& \leq C\left\|\omega^{n / 2 \pm 0} f\right\|\left\|\omega^{\sigma} u\right\|
\end{aligned}
$$

which holds for $|\sigma|<n / 2$.

Another consequence is the following lemma.

Lemma 2.3. Let $0<\sigma=\sigma_{1}+\sigma_{2}$ and $\sigma_{1} \vee \sigma_{2}<n / 2$. Then

$$
\left\|\omega^{\sigma-n / 2}(u v)\right\| \leq C\left\|\omega^{\sigma_{1}} u\right\|\left\|\omega^{\sigma_{2}} v\right\| .
$$

We shall also need some commutator estimates, which are most conveniently stated in terms of homogeneous Besov spaces $\dot{B}_{r, q}^{\sigma}$ [1]. In the applications, we shall use only the fact that $\dot{B}_{2,2}^{\sigma}=\dot{H}^{\sigma}$. The following lemma is an extension of Lemma 3.6 in [5] and may have independent interest. The proof will be given in Appendix A1. 
Lemma 2.4. Let $P_{i}, i=1,2$ be homogeneous derivative polynomials of degree $\alpha_{i}$ or $\omega^{\alpha_{i}}$ for $\alpha_{i} \geq 0$. Let $\lambda>0$. Then for any (sufficiently regular) functions $m, u$ and $v$ the following estimates hold.

$$
\begin{aligned}
& \left|<P_{1} u,\left[\omega^{\lambda}, m\right] P_{2} v>\right| \leq C\left\|m ; \dot{B}_{r_{0}, 2}^{\sigma_{0}} \cap \nabla^{-1} \omega^{1-\nu} L^{q_{0}}\right\|\left\|u ; \dot{B}_{r_{1}, 2}^{\sigma_{1}} \cap L^{q_{1}}\right\| \\
& \left\|v ; \dot{B}_{r_{2}, 2}^{\sigma_{2}} \cap L^{q_{2}}\right\|
\end{aligned}
$$

with $0 \leq \nu \leq 1,1 \leq r_{i}, q_{i} \leq \infty, 0 \leq i \leq 2$,

$$
\begin{gathered}
\delta\left(q_{0}\right)=\sigma_{0}+\delta\left(r_{0}\right)-\nu \quad, \quad \delta\left(q_{i}\right)=\sigma_{i}+\delta\left(r_{i}\right), i=1,2 . \\
\sum_{0 \leq i \leq 2} \sigma_{i}+\delta\left(r_{i}\right)=\lambda+\alpha_{1}+\alpha_{2}+n / 2 \\
\left\{\begin{array}{l}
\sigma_{0}+\left(\sigma_{1} \wedge \sigma_{2}\right) \geq \lambda+\alpha_{1}+\alpha_{2} \\
\sigma_{1}+\sigma_{2} \geq \lambda+\alpha_{1}+\alpha_{2}-\nu
\end{array}\right.
\end{gathered}
$$

where $\delta(r) \equiv n / 2-n / r$ and $\nabla^{-1} \omega^{1-\nu} L^{q}$ is the space of tempered distributions $m$ such that $\omega^{\nu-1} \nabla m \in L^{q}$.

Remark 2.1. The condition (2.6) implies that the various spaces occuring in the RHS of (2.5) are homogeneous under dilation, and the condition (2.7) is the global homogeneity condition of the estimate. More general, possibly non homogeneous, estimates can be derived by the same method (see Appendix A1).

We shall repeatedly use the estimate of $s=\nabla \varphi$ with $\varphi$ defined by (1.18)

$$
\left\|\nabla^{j} s\right\|_{\infty}+\left\|\omega^{n / 2} \nabla^{j} s\right\| \leq C\left\|\omega^{n / 2 \pm 0} \nabla^{j} s\right\| \leq C t^{\lambda_{j}-1}\left\|v_{0} ; H^{\rho}\right\|^{2}
$$

for $j=0,1$, where

$$
\lambda_{j}=\gamma-(1 / 2)[1+j+\gamma-2 \rho]_{+} .
$$

The first inequality in (2.9) follows from Lemma 2.1 and the second one from

$$
\left\|\omega^{n / 2 \pm 0} \nabla^{j} s\right\|=C t^{\gamma-1}\left\|\omega^{1+j+\gamma-n / 2 \pm 0} \chi_{L}\left|v_{0}\right|^{2}\right\|
$$

from the definition of $\chi_{L}$ and from Lemma 2.3. Note that up to an $\varepsilon$ in the case of equality

$$
\lambda_{0}=\gamma \wedge(1 / 2+\delta) \quad, \quad \lambda_{1}=\gamma \wedge \delta
$$


where $\delta=\rho-1+\gamma / 2$, so that $\lambda_{1}>0$ and $\lambda_{0}>1 / 2$ for $\gamma>1 / 2$ and $\rho>1-\gamma / 2$. The latter condition is the subcriticality condition mentioned in the introduction.

We shall also need some phase estimates. The following lemma is a variant of Lemma 3.3 in [5].

Lemma 2.5. Let $\varphi$ be a real function. Let $\sigma \geq 0$ and $1 \leq q, r \leq \infty$. Then the following estimate holds

$$
\left\|(\exp (i \varphi)-1) ; \dot{B}_{r, q}^{\sigma}\right\| \leq C\left\|\varphi ; \dot{B}_{r, q}^{\sigma}\right\|\left(1+\left\|\varphi ; \dot{B}_{\infty, \infty}^{0}\right\|\right)^{[\sigma]}
$$

where $[\sigma]$ is the integral part of $\sigma$.

An essential tool in this paper, as in [4] [5] [6], is an estimate which follows from a local conservation law for solutions of a suitable linear Schrödinger equation.

Lemma 2.6. Let $1 / 2<\rho<n / 2$, let $I$ be an interval, let $s \in L_{l o c}^{\infty}\left(I, L^{\infty} \cap \dot{H}^{n / 2}\right)$, $s$ real $\mathbb{I R}^{n}$ vector valued, and let $v \in \mathcal{C}\left(I, H^{\rho}\right)$ be a solution of the equation

$$
i \partial_{t} v+(1 / 2) \Delta_{s} v=V v
$$

in I for some real $V \in L_{l o c}^{\infty}\left(I, L^{\infty}\right)$. Then for any $t_{1}, t \in I, t_{1} \leq t$, one can write

$$
|v(t)|^{2}-\left|v\left(t_{1}\right)\right|^{2}=V_{1}\left(t_{1}, t\right)+V_{2}\left(t_{1}, t\right)
$$

where $V_{1}$ and $V_{2}$ satisfy the following estimates :

$$
\left\|\omega^{2 \sigma-2-n / 2} V_{1}\left(t_{1}, t\right)\right\| \leq C \int_{t_{1}}^{t} d t^{\prime}\left\|\omega^{\sigma} v\left(t^{\prime}\right)\right\|^{2}
$$

for $1 / 2<\sigma \leq \rho \wedge(1+n / 4)$, and

$$
\left\|\omega^{2 \sigma-1-n / 2} V_{2}\left(t_{1}, t\right)\right\| \leq C \int_{t_{1}}^{t} d t^{\prime}\left(\left\|s\left(t^{\prime}\right)\right\|_{\infty}+\left\|\omega^{n / 2} s\left(t^{\prime}\right)\right\|\right)\left\|\omega^{\sigma} v\left(t^{\prime}\right)\right\|^{2}
$$

for $0<\sigma \leq \rho$.

Sketch of proof. The formal conservation law

$$
\partial_{t}|v|^{2}=-\operatorname{Im} \bar{v} \Delta_{s} v=-\operatorname{Im} \bar{v} \Delta v+\nabla \cdot s|v|^{2}
$$


yields (2.13), where for any test function $\psi$ of the space variable

$$
\begin{gathered}
<V_{1}\left(t_{1}, t\right), \psi>=-(i / 2) \int_{t_{1}}^{t} d t^{\prime}<v\left(t^{\prime}\right),[\Delta, \psi] v\left(t^{\prime}\right)>, \\
<V_{2}\left(t_{1}, t\right), \psi>=-\int_{t_{1}}^{t} d t^{\prime}<s\left(t^{\prime}\right)\left|v\left(t^{\prime}\right)\right|^{2}, \nabla \psi>.
\end{gathered}
$$

By Lemma 2.4 with $\alpha_{i}=0, \lambda=2, r_{i}=2(0 \leq i \leq 2), \sigma_{1}=\sigma_{2}=\sigma$ and therefore $\sigma_{0}=n / 2+2-2 \sigma$, we obtain

$$
\left|<V_{1}\left(t_{1}, t\right), \psi>\right| \leq C\left\|\omega^{n / 2+2-2 \sigma} \psi\right\| \int_{t_{1}}^{t} d t^{\prime}\left\|\omega^{\sigma} v\left(t^{\prime}\right)\right\|^{2}
$$

for $1 / 2<\sigma \leq \rho \wedge(1+n / 4)$, from which (2.14) follows by duality.

In order to estimate $V_{2}$, we estimate

$$
\left\|\omega^{2 \sigma-n / 2} s|v|^{2}\right\| \leq C\left(\|s\|_{\infty}+\left\|\omega^{n / 2} s\right\|\right)\left\|\omega^{\sigma} v\right\|^{2}
$$

by (2.3) and Lemma 2.3. The estimate (2.15) then follows from (2.18) (2.20) by duality.

We next exploit the previous lemma in the relevant situation.

Lemma 2.7. Let $1 / 2<\rho<n / 2$. Let $v_{0} \in H^{\rho}$ and let $s=\nabla \varphi$ with $\varphi$ defined by (1.18). Let $I=(0, T]$ and let $v \in L^{\infty}\left(I, H^{\rho}\right) \cap \mathcal{C}\left([0, T], L^{2}\right)$ satisfy the equation (2.12) in I for some real $V \in L_{l o c}^{\infty}\left(I, L^{\infty}\right)$. Then $|v(t)|^{2}-|v(0)|^{2}$ tends to zero in $\dot{H}^{\mu}$ for $-1-n / 2<\mu<2 \rho-n / 2$ when $t$ tends to zero. Furthermore

$$
|v(t)|^{2}-|v(0)|^{2}=V_{1}(0, t)+V_{2}(0, t)
$$

with

$$
\left\|\omega^{2 \sigma-2-n / 2} V_{1}(0, t)\right\| \leq C a^{2} t
$$

for $1 / 2<\sigma \leq \rho \wedge(1+n / 4)$, and

$$
\left\|\omega^{2 \sigma-1-n / 2} V_{2}(0, t)\right\| \leq C a^{2} a_{0}^{2} t^{\lambda_{0}}
$$

for $0<\sigma \leq \rho$, with

$$
a=\left\|v ; L^{\infty}\left(I, H^{\rho}\right)\right\| \quad, \quad a_{0}=\left\|v_{0} ; H^{\rho}\right\| .
$$

Proof. We first prove that $V_{1}\left(t_{1}, t\right)$ and $V_{2}\left(t_{1}, t\right)$ defined by (2.17) (2.18) converge when $t_{1} \rightarrow 0$ in the norms occuring in (2.14) (2.15) and that the limits satisfy (2.22) 
(2.23). This is obvious for $V_{1}$. As regards $V_{2}$, we estimate $s$ in (2.15) by (2.9) with $j=0$. The resulting power of $t^{\prime}$ in the integral is then integrable at $t=0$ since $\lambda_{0}>0$. This proves the convergence of $V_{2}\left(t_{1}, t\right)$ as $t_{1} \rightarrow 0$ and the estimate (2.23).

On the other hand, by Lemma $2.3,|v(t)|^{2}$ is bounded in $\dot{H}^{2 \rho-n / 2}$ uniformly in $t$. Together with (2.13) and with the previous convergence of $V_{1}\left(t_{1}, t\right)$ and $V_{2}\left(t_{1}, t\right)$, this implies that $|v(t)|^{2}-\left|v\left(t_{1}\right)\right|^{2}$ converges in $\dot{H}^{\mu}$ for $-1-n / 2<\mu<2 \rho-n / 2$ when $t_{1}$ tends to zero for fixed $t$. We next identify the limit. Now

$$
|v(t)|^{2}-\left|v\left(t_{1}\right)\right|^{2}=|v(t)|^{2}-|v(0)|^{2}-\left(\left|v\left(t_{1}\right)\right|^{2}-|v(0)|^{2}\right)
$$

and from Lemma 2.3

$$
\left\|\omega^{\sigma-n / 2}\left(\left|v\left(t_{1}\right)\right|^{2}-|v(0)|^{2}\right)\right\| \leq C\left\|v\left(t_{1}\right)-v(0)\right\|\left\|\omega^{\sigma}\left(v\left(t_{1}\right)+v(0)\right)\right\|
$$

for $0<\sigma \leq \rho$, so that $|v(t)|^{2}-\left|v\left(t_{1}\right)\right|^{2}$ tends to $|v(t)|^{2}-|v(0)|^{2}$ in $\dot{H}^{\mu}$ for $-n / 2<\mu \leq$ $\rho-n / 2$ when $t_{1}$ tends to zero for fixed $t$. By an appropriate abstract argument, this implies that the same convergence holds in the whole range $-1-n / 2<\mu<2 \rho-n / 2$. This also implies (2.21), which together with the available estimates, completes the proof of the stated convergence.

Remark 2.2. The difference $|v(t)|^{2}-|v(0)|^{2}$ tends to zero in some norms which are not expected to be finite for $|v(t)|^{2}$ and $|v(0)|^{2}$ separately, typically in $\dot{H}^{\mu}$ for $-1-n / 2<\mu \leq-n / 2$.

Remark 2.3. In most of the applications, we shall take $v_{0}=v(0)$, but this is not needed in Lemma 2.7.

\section{The linearized Cauchy problem for $\mathrm{v}$}

In this section we study the Cauchy problem for the linearized equation (1.23) with $L(v)$ defined by (1.22) for a given $v$, with initial time $t_{0} \geq 0$. We first give a preliminary result with $t_{0}>0$, where we do not study the behaviour of the solution as $t$ tends to zero.

Proposition 3.1. Let $\rho>\gamma / 2$, let $I=(0, T]$, let $v_{0} \in H^{\rho}$ and let $v \in L_{l o c}^{\infty}\left(I, H^{\rho}\right)$. Let $0 \leq \rho^{\prime}<n / 2$, let $0<t_{0} \leq T$ and let $v_{0}^{\prime} \in H^{\rho^{\prime}}$. Then the equation (1.23) has a 
unique solution $v^{\prime} \in \mathcal{C}\left(I, H^{\rho^{\prime}}\right)$ with $v^{\prime}\left(t_{0}\right)=v_{0}^{\prime}$. The solution satisfies

$$
\left\|v^{\prime}(t)\right\|=\left\|v_{0}^{\prime}\right\|
$$

for all $t \in I$ and is unique in $\mathcal{C}\left(I, L^{2}\right)$.

The proof is sketched in Appendix A2.

We next study the boundedness and continuity properties near $t=0$ of the solutions of (1.23) obtained in Proposition 3.1. Since we shall eventually be interested in taking $\rho^{\prime}=\rho$, we already impose the condition $\rho<n / 2$ in the next proposition (see however Remark 3.2 below).

Proposition 3.2. Let $1-\gamma / 2<\rho<n / 2$, let $I=(0, T]$ and let $v \in L^{\infty}\left(I, H^{\rho}\right) \cap$ $\mathcal{C}\left([0, T], L^{2}\right)$ with $v(0)=v_{0}$. Let $s=\nabla \varphi$ with $\varphi$ defined by (1.18). Let $v$ satisfy the equation (2.12) in I for some real $V \in L_{\text {loc }}^{\infty}\left(I, L^{\infty}\right)$. Let $1 / 2 \leq \rho^{\prime}<n / 2$ and let $v^{\prime} \in \mathcal{C}\left(I, H^{\rho^{\prime}}\right)$ be a solution of the equation (1.23) in I. Then

(1) $v^{\prime} \in\left(\mathcal{C} \cap L^{\infty}\right)\left(I, H^{\rho^{\prime}}\right) \cap \mathcal{C}_{w}\left([0, T], H^{\rho^{\prime}}\right) \cap \mathcal{C}\left([0, T], H^{\sigma}\right)$ for $0 \leq \sigma<\rho^{\prime}$.

(2) For all $t \in[0, T], t_{1} \in I$, the following estimate holds

$$
\left\|\omega^{\rho^{\prime}} v^{\prime}(t)\right\| \leq\left\|\omega^{\rho^{\prime}} v^{\prime}\left(t_{1}\right)\right\| E\left(\left|t-t_{1}\right|\right)
$$

where

$$
\begin{aligned}
E(t) & \equiv E(t, a)=\exp \left\{C\left(a^{2} t^{\lambda_{1}}+a^{4} t^{2 \lambda_{0}-1}\right)\right\}, \\
a & =\left\|v ; L^{\infty}\left(I, H^{\rho}\right)\right\|
\end{aligned}
$$

and $\lambda_{j}$ is defined by (2.10).

(3) For all $t, t_{1} \in[0, T]$, the following estimate holds

$$
\left\|v^{\prime}(t)-v^{\prime}\left(t_{1}\right)\right\| \leq C\left|t-t_{1}\right|^{\left(\rho^{\prime} / 2\right) \wedge(2 \gamma-1)}\left(1+a^{2}\right)^{2}\left\|v^{\prime}\left(t_{1}\right) ; H^{\rho^{\prime}}\right\| .
$$

Remark 3.1. The estimate (3.1) for $t, t_{1} \in I$ holds for $0 \leq \rho^{\prime}<n / 2$, as will be clear from the proof. The condition $\rho^{\prime} \geq 1 / 2$ is needed to derive (3.4) which is used in turn to extend (3.1) to $t=0$.

Remark 3.2. The assumption $\rho<n / 2$ in Proposition 3.2 can be dispensed with at the expense of using slightly different estimates, which yield different powers of $t$ 
in (3.2) and (3.4).

Proof. We know already that the $L^{2}$ - norm of $v^{\prime}$ is conserved. The bulk of the proof consists in deriving the estimates (3.1) and (3.4) for $t, t_{1} \in I$. We begin with (3.1). From (1.22) (1.23) we obtain

$$
\begin{aligned}
\partial_{t}\left\|\omega^{\rho^{\prime}} v^{\prime}\right\|^{2} & =\operatorname{Im}<v^{\prime},\left[\omega^{2 \rho^{\prime}}, L(v)\right] v^{\prime}> \\
& =\operatorname{Re}<v^{\prime},\left[\omega^{2 \rho^{\prime}}, s\right] \cdot \nabla v^{\prime}>+\operatorname{Im}<v^{\prime},\left[\omega^{2 \rho^{\prime}}, f\right] v^{\prime}>
\end{aligned}
$$

where

$$
f=(1 / 2) s^{2}+t^{\gamma-2} g_{S}(v)+\left(t^{\gamma-2} g_{L}\left(v_{0}\right)-\partial_{t} \varphi\right)+t^{\gamma-2}\left(g_{L}(v)-g_{L}\left(v_{0}\right)\right)
$$

We estimate the first term in the RHS of (3.5) by Lemma 2.4 with $\lambda=2 \rho^{\prime}, \alpha_{1}=0$, $\alpha_{2}=1, r_{i}=2, \sigma_{1}=\sigma_{2}=\rho^{\prime}$, so that $\sigma_{0}=1+n / 2$ and $q_{0}=\infty$.

We estimate similarly the last term by Lemma 2.4 with $\lambda=2 \rho^{\prime}, \alpha_{1}=\alpha_{2}=0$, $r_{i}=2, \sigma_{1}=\sigma_{2}=\rho^{\prime}$, so that $\sigma_{0}=n / 2$ and $\delta\left(q_{0}\right)=n / 2-1$. We obtain

$$
\left|\partial_{t}\left\|\omega^{\rho^{\prime}} v^{\prime}\right\|^{2}\right| \leq C\left(\left\|\omega^{n / 2} \nabla s\right\|+\|\nabla s\|_{\infty}+\left\|\omega^{n / 2} f\right\|\right)\left\|\omega^{\rho^{\prime}} v^{\prime}\right\|^{2} .
$$

We estimate the various norms successively. We first estimate $\nabla s$ by (2.9) with $j=1$ so that

$$
\left\|\omega^{n / 2} \nabla s\right\|+\|\nabla s\|_{\infty} \leq C a^{2} t^{\lambda_{1}-1}
$$

and similarly

$$
\left\|\omega^{n / 2} s^{2}\right\| \leq\left\|\omega^{n / 2 \pm 0} s\right\|^{2} \leq C a^{4} t^{2 \lambda_{0}-2}
$$

by Lemma 2.1 and (2.9) with $j=0$.

We next estimate

$$
\begin{aligned}
t^{\gamma-2}\left\|\omega^{n / 2} g_{S}(v)\right\| & \leq C t^{\gamma-2+\rho-\gamma / 2}\left\|\omega^{\rho} v\right\|^{2} \\
& \leq C a^{2} t^{\lambda_{1}-1}
\end{aligned}
$$

for $\rho \geq \gamma / 2$, and similarly (see (1.19) )

$$
\begin{aligned}
& \left\|\omega^{n / 2}\left(\partial_{t} \varphi-t^{\gamma-2} g_{L}\left(v_{0}\right)\right)\right\|=(1-\gamma)^{-1} t^{\gamma-2}\left\|\omega^{n / 2} \tilde{\chi}_{L} g\left(v_{0}\right)\right\| \\
& \leq C t^{\gamma-2+\rho-\gamma / 2}\left\|\omega^{\rho} v_{0}\right\|^{2} \leq C a^{2} t^{\lambda_{1}-1} .
\end{aligned}
$$


The contribution of the last term in $f$ is estimated by the use of Lemma 2.7. From (2.21), from (2.22) with $\sigma=(1+\gamma / 2) \wedge \rho>1 / 2$ and from (2.23) with $\sigma=(1+\gamma) / 2 \wedge \rho>0$, we obtain

$$
\begin{aligned}
t^{\gamma-2}\left\|\omega^{n / 2}\left(g_{L}(v)-g_{L}\left(v_{0}\right)\right)\right\| & =C t^{\gamma-2}\left\|\omega^{\gamma-n / 2} \chi_{L}\left(V_{1}(0, t)+V_{2}(0, t)\right)\right\| \\
& \leq C\left(a^{2} t^{\lambda_{1}-1}+a^{4} t^{2 \lambda_{0}-2}\right) .
\end{aligned}
$$

Collecting (3.7)- (3.12), we obtain

$$
\left|\partial_{t}\left\|\omega^{\rho^{\prime}} v^{\prime}(t)\right\|^{2}\right| \leq N(t)\left\|\omega^{\rho^{\prime}} v^{\prime}(t)\right\|^{2}
$$

where

$$
N(t)=C\left(a^{2} t^{\lambda_{1}-1}+a^{4} t^{2 \lambda_{0}-2}\right) .
$$

The crucial point of this estimate is that $N(t)$ is integrable in time at $t=0$ since $\gamma>1 / 2$ and $\rho>1-\gamma / 2($ see (2.11) $)$. Note that

$$
\gamma / 2<1 / 2<1-\gamma / 2<(1+\gamma) / 2<1+\gamma / 2
$$

for $1 / 2<\gamma<1$. Therefore the condition $\rho>1-\gamma / 2$ implies the conditions $\rho \geq \gamma / 2$ and $\rho>1 / 2$ used in the proof of (3.10) and (3.12) respectively. Furthermore, there exists an interval, namely $1-\gamma / 2<\rho<(1+\gamma) / 2$ where the [ ]+ brackets are inactive. The estimate (3.1) (3.2) follows from (3.13) (3.14) by integration for $t_{1}$, $t \in I$.

We next derive the estimate (3.4) for $t, t_{1} \in I$. For that purpose we define (see (1.8) )

$$
\begin{gathered}
\widetilde{v}^{\prime}(t)=U(-t) v^{\prime}(t) \\
\widetilde{L}=L(v)+(1 / 2) \Delta=i s \cdot \nabla+(i / 2)(\nabla \cdot s)+f
\end{gathered}
$$

with $f$ given by (3.6). We rewrite (1.23) as

$$
i \partial_{t} \widetilde{v}^{\prime}=U(-t) \widetilde{L} U(t) \widetilde{v}^{\prime}
$$

so that for $t, t_{1} \in I$, for fixed $t_{1}$,

$$
\begin{aligned}
\partial_{t}\left\|\widetilde{v}^{\prime}(t)-\widetilde{v}^{\prime}\left(t_{1}\right)\right\|^{2} & =2 \operatorname{Im}<\widetilde{v}^{\prime}(t)-\widetilde{v}^{\prime}\left(t_{1}\right), U(-t) \widetilde{L} U(t) \widetilde{v}^{\prime}\left(t_{1}\right)> \\
& =2 \operatorname{Im}<w, \widetilde{L} v_{*}>
\end{aligned}
$$

where

$$
\left\{\begin{array}{l}
v_{*}=U\left(t-t_{1}\right) v^{\prime}\left(t_{1}\right) \\
w=v^{\prime}(t)-v_{*} .
\end{array}\right.
$$


We estimate

$$
\begin{aligned}
& \left|\partial_{t}\|w\|^{2}\right| \leq 2\left|\operatorname{Re}<w, s \cdot \nabla v_{*}>\right| \\
& +C\|w\|\left(\left\|\omega^{n / 2-\rho_{1}} \nabla \cdot s\right\|+\left\|\omega^{n / 2-\rho_{1}} f\right\|\right)\left\|\omega^{\rho_{1}} v^{\prime}\left(t_{1}\right)\right\|
\end{aligned}
$$

for some $\rho_{1}$ with $0<\rho_{1} \leq \rho^{\prime}$, to be chosen later.

For $0<\rho^{\prime}<1$, we write

$$
<w, s \cdot \nabla v_{*}>=-<\omega^{-\rho^{\prime}} \nabla \cdot s w, \omega^{\rho^{\prime}} v_{*}>
$$

and we estimate by Lemma 2.2

$$
\left|<w, s \cdot \nabla v_{*}>\right| \leq C\left\|\omega^{1-\rho^{\prime}} w\right\|\left(\|s\|_{\infty}+\left\|\omega^{n / 2} s\right\|\right)\left\|\omega^{\rho^{\prime}} v^{\prime}\left(t_{1}\right)\right\| .
$$

For $\rho^{\prime}=1$, we estimate

$$
\left|<w, s \cdot \nabla v_{*}>\right| \leq\|w\|\|s\|_{\infty}\left\|\omega^{\rho^{\prime}} v^{\prime}\left(t_{1}\right)\right\|
$$

For $\rho^{\prime}>1$, we estimate

$$
\left|<w, s \cdot \nabla v_{*}>\right| \leq C\|w\|\left\|\omega^{n / 2-\rho_{1}} \nabla s\right\|\left\|\omega^{\rho_{1}} v^{\prime}\left(t_{1}\right)\right\|
$$

for $1<\rho_{1} \leq \rho^{\prime}$.

Collecting (3.20)-(3.24) yields

$$
\begin{aligned}
& \left|\partial_{t}\|w\|^{2}\right| \leq C\left\{\chi\left(\rho^{\prime} \leq 1\right)\left\|\omega^{1-\rho^{\prime}} w\right\|\left(\|s\|_{\infty}+\left\|\omega^{n / 2} s\right\|\right)\left\|\omega^{\rho^{\prime}} v^{\prime}\left(t_{1}\right)\right\|\right. \\
& \left.+\|w\|\left(\left\|\omega^{n / 2-\rho_{1}} \nabla s\right\|+\left\|\omega^{n / 2-\rho_{1}} f\right\|\right)\left\|\omega^{\rho_{1}} v^{\prime}\left(t_{1}\right)\right\|\right\}
\end{aligned}
$$

with $0<\rho_{1} \leq \rho^{\prime}$ and $\rho_{1}>1$ in the $\nabla s$ term if $\rho^{\prime}>1$.

For $1 / 2 \leq \rho^{\prime} \leq 1$, we interpolate

$$
\left\|\omega^{1-\rho^{\prime}} w\right\| \leq y^{\theta}\left\|\omega^{\rho^{\prime}} w\right\|^{1 / \rho^{\prime}-1}
$$

where

$$
y=\|w(t)\|^{2} \quad, \quad \theta=1-1 /\left(2 \rho^{\prime}\right)
$$

so that (3.25) becomes

$$
\begin{aligned}
\left|\partial_{t} y\right| \leq & C\left\{\chi\left(\rho^{\prime} \leq 1\right)\left(\|s\|_{\infty}+\left\|\omega^{n / 2} s\right\|\right) a_{1}^{1 / \rho^{\prime}} y^{\theta}\right. \\
& \left.+\left(\left\|\omega^{n / 2-\rho_{1}} \nabla s\right\|+\left\|\omega^{n / 2-\rho_{1}} f\right\|\right) a_{1}^{\prime} y^{1 / 2}\right\}
\end{aligned}
$$


with $a_{1}^{\prime}=\left\|v^{\prime}\left(t_{1}\right) ; H^{\rho^{\prime}}\right\|$. We estimate $s$ in the first term in the RHS of (3.26) by (2.9) with $j=0$ and we estimate the various contributions to the second term for suitable values of $\rho_{1}$. We first estimate

$$
\begin{aligned}
\left\|\omega^{n / 2-\rho_{1}} \nabla s\right\| & \leq C t^{\gamma-1}\left\|\omega^{2+\gamma-\rho_{1}-n / 2} \chi_{L}\left|v_{0}\right|^{2}\right\| \\
& \leq C t^{\gamma-1}\left\|\omega^{\rho_{2}} v_{0}\right\|^{2}
\end{aligned}
$$

by Lemma 2.3 with $0<\rho_{1} \leq \rho^{\prime}, 0<\rho_{2} \leq \rho, \rho_{1}>1$ if $\rho^{\prime}>1$ and $\rho_{1}+2 \rho_{2}=2+\gamma$, in the case where $\rho^{\prime}+2 \rho \geq 2+\gamma$. In the opposite case, we take $\rho_{1}=\rho^{\prime}, \rho_{2}=\rho$, and we use the cut off $\chi_{L}$ so that finally, for the relevant choice of $\rho_{1}$,

$$
\left\|\omega^{n / 2-\rho_{1}} \nabla s\right\| \leq C a^{2} t^{\mu_{1}-1}
$$

with

$$
\mu_{1}=\gamma-(1 / 2)\left(2+\gamma-2 \rho-\rho^{\prime}\right)_{+}
$$

Similarly, we estimate

$$
\begin{aligned}
& \left\|\omega^{n / 2-\rho_{1}}|s|^{2}\right\| \leq C\left\|\omega^{n / 2-\rho_{1} / 2} s\right\|^{2} \\
& =C t^{2 \gamma-2}\left\|\omega^{1+\gamma-\rho_{1} / 2-n / 2} \chi_{L}\left|v_{0}\right|^{2}\right\|^{2} \leq C a^{4} t^{2 \mu_{0}-2}
\end{aligned}
$$

with

$$
\mu_{0}=\gamma-(1 / 2)\left(1+\gamma-2 \rho-\rho^{\prime} / 2\right)_{+}
$$

We next estimate, with $\rho_{1}=\rho^{\prime}$

$$
\begin{aligned}
& t^{\gamma-2}\left\|\omega^{n / 2-\rho^{\prime}} g_{S}(v)\right\| \leq C a^{2} t^{\mu_{1}-1} \\
& \left\|\omega^{n / 2-\rho^{\prime}}\left(\partial_{t} \varphi-t^{\gamma-2} g_{L}\left(v_{0}\right)\right)\right\| \leq C a^{2} t^{\mu_{1}-1} .
\end{aligned}
$$

We next consider

$$
t^{\gamma-2}\left\|\omega^{n / 2-\rho_{1}}\left(g_{L}(v)-g_{L}\left(v_{0}\right)\right)\right\|=C t^{\gamma-2}\left\|\omega^{\gamma-\rho_{1}-n / 2} \chi_{L}\left(V_{1}+V_{2}\right)\right\|
$$

where $V_{i} \equiv V_{i}(0, t), i=1,2$, are defined by (2.17) (2.18). By (2.14) we estimate

$$
\left\|\omega^{\gamma-\rho_{1}-n / 2} \chi_{L} V_{1}\right\| \leq C \int_{0}^{t} d t^{\prime}\left\|\omega^{\rho_{2}} v\left(t^{\prime}\right)\right\|^{2}
$$

for $0<\rho_{1} \leq \rho^{\prime}, 1 / 2<\rho_{2} \leq \rho$ and $\rho_{1}+2 \rho_{2}=2+\gamma$ in the case where $\rho^{\prime}+2 \rho \geq 2+\gamma$. In the opposite case, we take $\rho_{1}=\rho^{\prime}, \rho_{2}=\rho$ and we use the cut off $\chi_{L}$, so that finally, for the relevant choice of $\rho_{1}$,

$$
t^{\gamma-2}\left\|\omega^{\gamma-\rho_{1}-n / 2} \chi_{L} V_{1}\right\| \leq C a^{2} t^{\mu_{1}-1}
$$


We next estimate

$$
\begin{aligned}
& \left\|\omega^{\gamma-\rho_{1}-n / 2} \chi_{L} V_{2}\right\| \leq \int_{0}^{t} d t^{\prime}\left\|\omega^{1+\gamma-\rho_{1}-n / 2} \chi_{L}(t)\left(s|v|^{2}\right)\left(t^{\prime}\right)\right\| \\
& \leq C \int_{0}^{t} d t^{\prime}\left\|\omega^{1+\gamma-\rho_{1} / 2-n / 2}\left|v\left(t^{\prime}\right)\right|^{2}\right\|\left\|\omega^{n / 2-\rho_{1} / 2} s\left(t^{\prime}\right)\right\| \\
& \leq C \int_{0}^{t} d t^{\prime} t^{\prime \gamma-1}\left\|\omega^{\rho_{2}} v\left(t^{\prime}\right)\right\|^{2}\left\|\omega^{1+\gamma-\rho_{1} / 2-n / 2} \chi_{L}\left(t^{\prime}\right)\left|v_{0}\right|^{2}\right\| \\
& \leq C\left\|\omega^{\rho_{2}} v_{0}\right\|^{2} \int_{0}^{t} d t^{\prime} t^{\prime \gamma-1}\left\|\omega^{\rho_{2}} v\left(t^{\prime}\right)\right\|^{2}
\end{aligned}
$$

by repeated use of Lemma 2.3, for $0<\rho_{1} \leq \rho^{\prime}, \rho_{1}<1+\gamma, 0<\rho_{2} \leq \rho$ and $\rho_{1} / 2+2 \rho_{2}=1+\gamma$, in the case where $\rho^{\prime} / 2+2 \rho \geq 1+\gamma$. In the opposite case, we take $\rho_{1}=\rho^{\prime}, \rho_{2}=\rho$ and we use the cut offs $\chi_{L}(t)$ and $\chi_{L}\left(t^{\prime}\right)$, so that finally, for the relevant choice of $\rho_{1}$,

$$
\begin{aligned}
& t^{\gamma-2}\left\|\omega^{\gamma-\rho_{1}-n / 2} \chi_{L} V_{2}\right\| \leq C\left\|v_{0} ; H^{\rho}\right\|^{2} t^{\mu_{0}-2} \\
& \times \int_{0}^{t} d t^{\prime} t^{\prime \mu_{0}-1}\left\|v\left(t^{\prime}\right) ; H^{\rho}\right\|^{2} \leq C a^{4} t^{2 \mu_{0}-2} .
\end{aligned}
$$

(Note that in the second case

$$
\left.\rho^{\prime}<2+2 \gamma-4 \rho<4 \gamma-2 \leq 1+\gamma \quad \text { for } \gamma \leq 1\right)
$$

Collecting (3.26), (2.9) with $j=0$ and (3.28) (3.30) (3.32) (3.33) (3.3.35) yields

$$
\begin{aligned}
\left|\partial_{t} y\right| \leq & C\left\{\chi\left(\rho^{\prime} \leq 1\right) a^{2} t^{\lambda_{0}-1} a_{1}^{1 / \rho^{\prime}} y^{\theta}\right. \\
& \left.+\left(a^{2} t^{\mu_{1}-1}+a^{4} t^{2 \mu_{0}-2}\right) a_{1}^{\prime} y^{1 / 2}\right\} .
\end{aligned}
$$

Using the fact that the differential inequality

$$
\left|\partial_{t} y\right| \leq \sum_{i} b_{i} t^{\nu_{i}-1} y^{\theta_{i}}
$$

with $0 \leq \theta_{i}<1, \nu_{i}>0$ implies

$$
y(t) \leq C \sum_{i}\left(b_{i} \nu_{i}^{-1}\left|t^{\nu_{i}}-t_{1}^{\nu_{i}}\right|\right)^{1 /\left(1-\theta_{i}\right)}
$$

for $t, t_{1}>0$ and $y\left(t_{1}\right)=0$, we obtain

$$
\begin{aligned}
\|w\| & \leq C\left\{\chi\left(\rho^{\prime} \leq 1\right) a^{2 \rho^{\prime}}\left|t-t_{1}\right|^{\rho^{\prime} \lambda_{0}}+a^{2}\left|t-t_{1}\right|^{\mu_{1}}+a^{4}\left|t-t_{1}\right|^{2 \mu_{0}-1}\right\} a_{1}^{\prime} \\
& \leq C\left(\chi\left(\rho^{\prime} \leq 1\right) a^{2 \rho^{\prime}}+a^{2}\left(1+a^{2}\right)\right)\left|t-t_{1}\right|^{\mu} a_{1}^{\prime} .
\end{aligned}
$$


where

$$
\begin{aligned}
\mu & =\rho^{\prime} \lambda_{0} \wedge \mu_{1} \wedge\left(2 \mu_{0}-1\right) \\
& =\rho^{\prime} \gamma \wedge \rho^{\prime}(1 / 2+\delta) \wedge \gamma \wedge\left(\rho^{\prime} / 2+\delta\right) \wedge(2 \gamma-1) \wedge\left(\rho^{\prime} / 2+2 \delta\right) \\
& \geq \rho^{\prime} / 2 \wedge(2 \gamma-1)
\end{aligned}
$$

since $1 / 2<\gamma<1$ and $\delta \equiv \rho-1+\gamma / 2>0$.

On the other hand, we estimate

$$
\begin{aligned}
& \left\|v^{\prime}(t)-v^{\prime}\left(t_{1}\right)\right\| \leq\|w\|+\left\|\left(U\left(t-t_{1}\right)-1\right) v^{\prime}\left(t_{1}\right)\right\| \\
& \leq\|w\|+\left|t-t_{1}\right|^{\left(\rho^{\prime} / 2\right) \wedge 1}\left\|\omega^{\rho^{\prime} \wedge 2} v^{\prime}\left(t_{1}\right)\right\| .
\end{aligned}
$$

Collecting (3.39) (3.41) yields (3.4) for $t, t_{1} \in I$.

We now exploit (3.1) and (3.4) in $I$ to complete the proof of the proposition. From (3.1) it follows that $v^{\prime} \in L^{\infty}\left(I, H^{\rho^{\prime}}\right)$. From (3.1) and (3.4) it then follows that $v^{\prime}$ has a limit $v^{\prime}(0)$ in $L^{2}$ and that (3.4) holds for $t, t_{1} \in[0, T]$. It then follows by a standard abstract argument that $v^{\prime}(0) \in H^{\rho^{\prime}}$, that $v^{\prime} \in \mathcal{C}_{w}\left([0, T], H^{\rho^{\prime}}\right) \cap \mathcal{C}\left([0, T], H^{\sigma}\right)$ for $0 \leq \sigma<\rho^{\prime}$, and that (3.1) holds for all $t \in[0, T], t_{1} \in I$.

We have not proved so far that $v^{\prime} \in \mathcal{C}\left([0, T], H^{\rho^{\prime}}\right)$. This is true but requires a separate argument.

Proposition 3.3. Under the assumptions of Proposition 3.2, $v^{\prime} \in \mathcal{C}\left([0, T], H^{\rho^{\prime}}\right)$ and (3.1) holds for all $t, t_{1} \in[0, T]$.

The proof is identical with that of Proposition 3.3 of [4.

We can now state the main result on the Cauchy problem for the linearized equation (1.23).

Proposition 3.4. Let $1-\gamma / 2<\rho<n / 2$. Let $I=(0, T]$ and let $v \in L^{\infty}\left(I, H^{\rho}\right) \cap$ $\mathcal{C}\left([0, T], L^{2}\right)$ with $v(0)=v_{0}$. Let $s=\nabla \varphi$ with $\varphi$ defined by (1.18). Let $v$ satisfy the equation (2.12) in I for some real $V \in L_{\text {loc }}^{\infty}\left(I, L^{\infty}\right)$. Let $1 / 2<\rho^{\prime}<n / 2$ and let $v_{0}^{\prime} \in H^{\rho^{\prime}}$. Let $t_{0} \in[0, T]$. Then there exists a unique solution $v^{\prime} \in \mathcal{C}\left([0, T], H^{\rho^{\prime}}\right)$ of the equation (1.23) with $v^{\prime}(0)=v_{0}^{\prime}$. Furthermore $v^{\prime}$ satisfies the estimates (3.1) and (3.4) for all $t, t_{1} \in[0, T]$. The solution is actually unique in $\mathcal{C}\left([0, T], L^{2}\right)$. 
Proof. For $t_{0}>0$, the result follows from Propositions 3.1, 3.2 and 3.3. For $t_{0}=0$, it will be proved by a limiting procedure on $t_{0}$. For any $t_{1} \in I$, let $v_{t_{1}}^{\prime}$ be the solution of (1.23) with $v_{t_{1}}^{\prime}\left(t_{1}\right)=v_{0}^{\prime}$ given by Propositions 3.1 and 3.2. Let now $0<t_{1}<t_{2} \leq T$. It follows from (3.1) that

$$
\left\|\omega^{\rho^{\prime}} v_{t_{i}}^{\prime}(t)\right\| \leq E\left(\left|t-t_{i}\right|\right)\left\|\omega^{\rho^{\prime}} v_{0}^{\prime}\right\|
$$

for $i=1,2$ and for all $t \in[0, T]$. Furthermore, from (3.4) and (3.42) and from $L^{2}$-norm conservation, it follows that

$$
\begin{aligned}
& \left\|v_{t_{2}}^{\prime}(t)-v_{t_{1}}^{\prime}(t)\right\|=\left\|v_{t_{2}}^{\prime}\left(t_{1}\right)-v_{0}^{\prime}\right\|=\left\|v_{t_{2}}^{\prime}\left(t_{1}\right)-v_{t_{2}}^{\prime}\left(t_{2}\right)\right\| \\
& \leq C\left|t_{2}-t_{1}\right|^{\left(\rho^{\prime} / 2\right) \wedge(2 \gamma-1)}\left(1+a^{2}\right)^{2}\left\|v_{0}^{\prime} ; H^{\rho^{\prime}}\right\| .
\end{aligned}
$$

From (3.43) it follows that $v_{t_{1}}^{\prime}$ converges in $L^{\infty}\left(I, L^{2}\right)$-norm to some $v^{\prime} \in \mathcal{C}\left([0, T], L^{2}\right)$ when $t_{1} \rightarrow 0$. From the uniform estimate (3.42) it follows by abstract arguments that $v^{\prime} \in\left(\mathcal{C}_{w} \cap L^{\infty}\right)\left([0, T], H^{\rho^{\prime}}\right) \cap \mathcal{C}\left([0, T], H^{\sigma}\right)$ for $0 \leq \sigma<\rho^{\prime}$, that $v^{\prime}$ satisfies the estimates of Proposition 3.2 and that $v^{\prime}(0)=v_{0}^{\prime}$. Furthermore $v^{\prime}$ is easily seen to satisfy (1.23) in $I$, so that $v^{\prime} \in \mathcal{C}\left(I, H^{\rho^{\prime}}\right)$. It remains to be proved that actually $v^{\prime}$ is strongly continuous in $H^{\rho^{\prime}}$ at $t=0$. This follows from Proposition 3.3, which has not been used so far. Alternatively it follows from the estimate (3.42) with $t_{1}=0$ that

$$
\lim _{t \rightarrow 0} \sup \left\|\omega^{\rho^{\prime}} v^{\prime}(t)\right\| \leq\left\|\omega^{\rho^{\prime}} v_{0}^{\prime}\right\| E(0)=\left\|\omega^{\rho^{\prime}} v^{\prime}(0)\right\|
$$

which together with weak continuity implies strong continuity at $t=0$.

Remark 3.3. Note that in the case where $t_{0}=0$, Proposition 3.3 is not needed for the proof of Proposition 3.4.

\section{The nonlinear Cauchy problem at time zero for $\mathbf{v}$ and $\mathbf{u}_{\mathbf{c}}$}

In this section we prove that the nonlinear equation (1.21) for $v$ with initial data at time $t_{0}$ has a unique solution in a small time interval. We then rewrite that result in terms of $u_{c}$, related to $v$ by (1.12), and we give some additional bounds and regularity properties for $u_{c}$. In order to solve the equation (1.21) for $v$, we show that the map $\Gamma: v \rightarrow v^{\prime}$ defined by Proposition 3.4 with $t_{0}=0$ is a contraction. For 
that purpose, we need to estimate the difference of two solutions of the linearized equation (1.23). For any pair of functions or operators $\left(f_{1}, f_{2}\right)$, we define

$$
f_{ \pm}=(1 / 2)\left(f_{2} \pm f_{1}\right)
$$

Lemma 4.1. Let $1-\gamma / 2<\rho<n / 2$. Let $I=(0, T]$ and let $v_{i}, i=1,2$ satisfy the assumptions of Proposition 3.4 with $v_{i}(0)=v_{0}$. Let $1 / 2<\rho^{\prime}<n / 2$ and let $v_{i}^{\prime}$, $i=1,2$ be the solutions of the equation (1.23) with $v_{i}^{\prime}(0)=v_{0}^{\prime} \in H^{\rho^{\prime}}$ obtained in Proposition 3.4. Then the following estimate holds for all $t, 0<t \leq T$ :

$$
\left\|v_{-}^{\prime} ; L^{\infty}\left((0, t], H^{\rho^{\prime}}\right)\right\| \leq C E(t, a) a a^{\prime}\left(t^{\lambda_{1}}+a^{2} t^{2 \lambda_{0}-1}\right)\left\|v_{-} ; L^{\infty}\left((0, t], H^{\rho}\right)\right\|
$$

where $E(t, a)$ is defined by (3.2) and

$$
a=\operatorname{Max}\left\|v_{i} ; L^{\infty}\left(I, H^{\rho}\right)\right\|, \quad a^{\prime}=\operatorname{Max}\left\|v_{i}^{\prime} ; L^{\infty}\left(I, H^{\rho^{\prime}}\right)\right\| .
$$

Proof. From (1.23) we obtain

$$
i \partial_{t} v_{-}^{\prime}=L_{2} v_{-}^{\prime}+L_{-} v_{1}^{\prime}
$$

where $L_{i}=L\left(v_{i}\right), g_{i}=g\left(v_{i}\right)$, so that

$$
L_{-}=t^{\gamma-2} g_{-}
$$

We estimate for $0 \leq \sigma \leq \rho^{\prime}$

$$
\partial_{t}\left\|\omega^{\sigma} v_{-}^{\prime}\right\|^{2}=2 \operatorname{Im}\left(<\omega^{\sigma} v_{-}^{\prime}, \omega^{\sigma} L_{2} v_{-}^{\prime}>+<\omega^{\sigma} v_{-}^{\prime}, \omega^{\sigma} L_{-} v_{1}^{\prime}>\right) .
$$

By the estimates in the proof of Proposition 3.2 (see in particular (3.1) ; see also Remark 3.1), we obtain

$$
\left\|\omega^{\sigma} v_{-}^{\prime}(t)\right\| \leq E(t, a) \int_{0}^{t} d t^{\prime} t^{\prime \gamma-2}\left\|\omega^{\sigma} g_{-} v_{1}^{\prime}\left(t^{\prime}\right)\right\|
$$

We next estimate

$$
\begin{aligned}
& \left\|\omega^{\sigma} g_{-} v_{1}^{\prime}\right\| \leq C\left\|\omega^{n / 2 \pm 0} g_{-}\right\|\left\|\omega^{\sigma} v_{1}^{\prime}\right\|, \\
& \left\|\omega^{n / 2 \pm 0} g_{S-}\right\| \leq C t^{\rho-\gamma / 2}\left\|\omega^{\rho} v_{-}\right\|\left\|\omega^{\rho} v_{+}\right\| .
\end{aligned}
$$

In order to estimate $g_{L-}$, we use again Lemmas 2.6 and 2.7. From the conservation law (2.16) and from the fact that $v_{-}(0)=0$ we obtain (see (2.21) $)$

$$
\left(|v(t)|^{2}\right)_{-}=V_{1-}(0, t)+V_{2-}(0, t)
$$


where (see (2.17) (2.18))

$$
\begin{aligned}
& V_{1-}(0, t)=-\int_{0}^{t} d t^{\prime} \operatorname{Im}\left(\bar{v}_{+} \Delta v_{-}+\bar{v}_{-} \Delta v_{+}\right)\left(t^{\prime}\right), \\
& V_{2-}(0, t)=\int_{0}^{t} d t^{\prime} \nabla \cdot\left(2 s \operatorname{Re} \bar{v}_{+} v_{-}\right)\left(t^{\prime}\right) .
\end{aligned}
$$

By the same estimates as in Lemma 2.6, we obtain

$$
\left\|\omega^{2 \sigma-2-n / 2} V_{1-}(0, t)\right\| \leq C \int_{0}^{t} d t^{\prime}\left(\left\|\omega^{\sigma} v_{+}\right\|\left\|\omega^{\sigma} v_{-}\right\|\right)\left(t^{\prime}\right)
$$

for $1 / 2<\sigma \leq \rho \wedge(1+n / 4)$,

$$
\left\|\omega^{2 \sigma-1-n / 2} V_{2-}(0, t)\right\| \leq C \int_{0}^{t} d t^{\prime}\left(\left(\|s\|_{\infty}+\left\|\omega^{n / 2} s\right\|\right)\left\|\omega^{\sigma} v_{+}\right\|\left\|\omega^{\sigma} v_{-}\right\|\right)\left(t^{\prime}\right)
$$

for $0<\sigma \leq \rho$. In the same way as in Proposition 3.2 (see especially (3.12)), we obtain

$$
t^{\prime \gamma-2}\left\|\omega^{\sigma} g_{-} v_{1}^{\prime}\right\| \leq C a a^{\prime}\left(t^{\prime \lambda_{1}-1}+a^{2} t^{\prime 2 \lambda_{0}-2}\right)\left\|v_{-} ; L^{\infty}\left(\left(0, t^{\prime}\right], H^{\rho}\right)\right\| .
$$

Substituting (4.10) into (4.4) yields (4.1).

We can now state the main result on the Cauchy problem at time zero for the equation (1.21).

Proposition 4.1. Let $1-\gamma / 2<\rho<n / 2$, let $v_{0} \in H^{\rho}$ and define $\varphi$ by (1.18). Then there exists $T>0$ and there exists a unique solution $v \in \mathcal{C}\left([0, T], H^{\rho}\right)$ of the equation (1.21) with $v(0)=v_{0}$. One can ensure that

$$
\begin{aligned}
& \left\|v ; L^{\infty}\left([0, T], H^{\rho}\right)\right\| \leq R=2\left\|v_{0} ; H^{\rho}\right\| \\
& C R^{2}\left(T^{\lambda_{1}}+R^{2} T^{2 \lambda_{0}-1}\right)=1
\end{aligned}
$$

for some $C$ independent of $v_{0}$.

Proof. Let $T>0$. Let $F\left(T, v_{0}\right)$ be the set of $v \in \mathcal{C}\left([0, T], H^{\rho}\right)$ such that $v(0)=v_{0}$ and satisfying the equation (2.12) in $(0, T]$ for some real $V \in L_{l o c}^{\infty}\left((0, T], L^{\infty}\right)$. It follows from Proposition 3.4 that $F\left(T, v_{0}\right)$ is stable under the map $\Gamma: v \rightarrow v^{\prime}$ defined by that proposition with $t_{0}=0$ and $v_{0}^{\prime}=v_{0}$. In fact, $v^{\prime}$ satisfies the equation (2.12) with

$$
V=t^{\gamma-2} g(v)-\partial_{t} \varphi \in L_{l o c}^{\infty}\left((0, T], L^{\infty}\right)
$$


Let $B(R)$ be the ball of radius $R$ in $\mathcal{C}\left([0, T], H^{\rho}\right)$. From Proposition 3.2 it follows that $B(R) \cap F\left(T, v_{0}\right)$ is stable under $\Gamma$ if

$$
E(T, R) \leq 2
$$

with $R=2\left\|v_{0} ; H^{\rho}\right\|$. Furthermore by Lemma 4.1, $\Gamma$ is a contraction in the $\left.L^{\infty}([0, T]), H^{\rho}\right)$-norm on that set under the condition (4.12) for a suitable $C$. Such a condition at the same time implies (4.13). Therefore for $T$ sufficiently small to satisfy (4.12), the map $\Gamma$ has a unique fixed point in $B(R)$ provided $F\left(T, v_{0}\right)$ is non empty. That set is not empty because it contains the solution of the linear equation (2.12) with $v(0)=v_{0}$ and $V=0$, obtained by a simplified version of Proposition 3.4. Clearly the fixed point $v$ satisfies the equation (1.21) and therefore belongs to $F\left(T, v_{0}\right)$.

We finally translate the main result of Proposition 4.1 in terms of $u_{c}$ and we derive additional bounds and regularity properties for $u_{c}$.

Proposition 4.2. Let $1-\gamma / 2<\rho<n / 2$, let $v_{0} \in H^{\rho}$ and define $\varphi$ by (1.18). Then there exists $T>0$ and there exists a unique solution $u_{c} \in \mathcal{C}\left([0, T], H^{\rho}\right)$ of the equation (1.11) such that $v$ defined by (1.12) satisfies the equation (1.21) with $v(0)=v_{0}$. Furthermore $u_{c}$ satisfies the estimate

$$
\left\|u_{c}(t) ; H^{\rho}\right\| \leq C a_{0}\left(1+a_{0}^{2} t^{\gamma-1}\right)^{1+[\rho]}
$$

for all $t \in(0, T]$, where $[\rho]$ is the integral part of $\rho$ and

$$
a_{0}=\left\|v_{0} ; H^{\rho}\right\| \text {. }
$$

Proof. The first statement follows from Proposition 4.1, except for the continuity of $u_{c}$. We first prove the estimate (4.14). By Lemmas 2.1 and 2.2 we estimate

$$
\left.\| \omega^{\rho} \exp (-i \varphi) v\right)\left\|\leq C\left(1+\left\|\omega^{\rho}(\exp (-i \varphi)-1)\right\|_{n / \rho}\right)\right\| \omega^{\rho} v \| .
$$

It follows from Lemma 2.5 that for $0<\rho<n / 2$

$$
\begin{aligned}
& \left\|\omega^{\rho}(\exp (-i \varphi)-1)\right\|_{n / \rho} \leq C\left\|\exp (-i \varphi)-1 ; \dot{B}_{n / \rho, 2}^{\rho}\right\| \\
& \leq C\left\|\varphi ; \dot{B}_{n / \rho, 2}^{\rho}\right\|\left(1+\left\|\varphi ; \dot{B}_{\infty, \infty}^{0}\right\|\right)^{[\rho]} \\
& \leq C\left\|\omega^{n / 2} \varphi\right\|\left(1+\left\|\omega^{n / 2} \varphi\right\|\right)^{[\rho]} .
\end{aligned}
$$


Using (1.18) and Lemma 2.3, we estimate

$$
\left\|\omega^{n / 2} \varphi\right\| \leq C t^{\gamma-1}\left\|\omega^{\gamma / 2} v_{0}\right\|^{2} \leq C a_{0}^{2} t^{\gamma-1}
$$

which together with (4.15) and (4.16) implies (4.14).

It remains to prove the continuity in time of $u_{c}$ in $H^{\rho}$. For that purpose it suffices to show that the multiplication by $\exp (-i \varphi)$ is strongly continuous in $t$ as an operator in $H^{\rho}$. Now for fixed $v$ and $t_{0}$

$$
\begin{aligned}
& \left\|\left(\exp (-i \varphi(t))-\exp \left(-i \varphi\left(t_{0}\right)\right)\right) v ; H^{\rho}\right\| \\
& \leq C\left(\|\delta \varphi\|_{\infty}+\left\|\omega^{n / 2} \delta \varphi\right\|\left(1+\left\|\omega^{n / 2} \delta \varphi\right\|\right)^{[\rho]}\right)\left\|\exp \left(-i \varphi\left(t_{0}\right)\right) v ; H^{\rho}\right\|
\end{aligned}
$$

where $\delta \varphi=\varphi(t)-\varphi\left(t_{0}\right)$ and it suffices to prove that $\varphi(t)$ is a continuous function of $t$ in $L^{\infty} \cap \dot{H}^{n / 2}$. This follows immediately from estimates similar to (4.17).

\section{Continuity with respect to initial data}

In this section we prove that the map $v_{0} \rightarrow v$ defined by Proposition 4.1 is continuous in the natural norms and that the map $v_{0} \rightarrow u_{c}$ defined by Proposition 4.2 satisfies similar continuity properties. As mentioned in the introduction, the proof of those properties given here requires the additional condition $\rho>3 / 4$, which is stronger than the condition $\rho>1-\gamma / 2$ for $\gamma>1 / 2$. We need to estimate the difference of two solutions of the linearized equation (1.23) corresponding to two functions $v_{1}$ and $v_{2}$ not necessarily satisfying the condition $v_{1}(0)=v_{2}(0)$. The following lemma is an extension of Lemma 4.1 where we drop that condition. Furthermore we assume for simplicity that $\rho^{\prime}=\rho$.

Lemma 5.1. Let $3 / 4<\rho<n / 2$. Let $I=(0, T]$ and let $v_{i}, i=1,2$, satisfy the assumptions of Proposition 3.4 with $v_{i}(0)=v_{0 i} \in H^{\rho}$. Let $v_{i}^{\prime}, i=1,2$ be the solutions of the equation (1.23) with $v_{i}^{\prime}(0)=v_{0 i}^{\prime} \in H^{\rho}$ obtained in Proposition 3.4. Let $0 \leq \sigma \leq \rho-1 / 2$ with $\rho+\sigma>1$. Define

$$
\begin{aligned}
& y \equiv y(t)=\left\|\omega^{\sigma} v_{-}(t)\right\|^{2}+\left\|v_{-}(t)\right\|^{2} \approx\left\|v_{-}(t) ; H^{\sigma}\right\|^{2} \\
& y^{\prime} \equiv y^{\prime}(t)=\left\|\omega^{\sigma} v_{-}^{\prime}(t)\right\|^{2}+\left\|v_{-}^{\prime}(t)\right\|^{2} \approx\left\|v_{-}^{\prime}(t) ; H^{\sigma}\right\|^{2}
\end{aligned}
$$


and $y_{0}=y(0)$. Then the following estimate holds

$$
\begin{aligned}
& \left|\partial_{t} y^{\prime}\right| \leq C t^{\lambda_{1}-1}\left(a^{2} y^{\prime}+a a^{\prime 2} y_{0}^{1 / 2}+a a^{\prime} y^{\prime / 2}\left(y_{0}^{1 / 2}+y^{1 / 2}+t^{-1} \int_{0}^{t} d t^{\prime} y^{1 / 2}\left(t^{\prime}\right)\right)\right) \\
& +C t^{2 \lambda_{0}-2}\left(a^{4} y^{\prime}+a^{3} a^{\prime} y^{\prime 1 / 2}\left(y_{0}^{1 / 2}+t^{-\lambda_{0}} \int_{0}^{t} d t^{\prime} t^{\prime \lambda_{0}-1} y^{1 / 2}\left(t^{\prime}\right)\right)\right)
\end{aligned}
$$

for all $t \in I$, where $\lambda_{0}, \lambda_{1}$ are defined by (2.10), and $a$, $a^{\prime}$ are defined by (4.2).

Proof. In the same way as in the proof of Lemma 4.1, we obtain from (1.23)

$$
i \partial_{t} v_{-}^{\prime}=L_{2} v_{-}^{\prime}+L_{-} v_{1}^{\prime}
$$

where $L_{i}=L\left(v_{i}\right)$,

$$
L_{-}=i s_{-} \cdot \nabla+(i / 2)\left(\nabla \cdot s_{-}\right)+f_{-}
$$

and $f$ is defined by (3.6). We estimate for $0 \leq \sigma \leq \rho$

$$
\partial_{t}\left\|\omega^{\sigma} v_{-}^{\prime}\right\|^{2}=2 \operatorname{Im}\left(<\omega^{\sigma} v_{-}^{\prime}, \omega^{\sigma} L_{2} v_{-}^{\prime}>+<\omega^{\sigma} v_{-}^{\prime}, \omega^{\sigma} L_{-} v_{1}^{\prime}>\right) .
$$

We estimate the first scalar product in (5.4) as in the proof of Proposition 3.2 namely $(\operatorname{see}(3.13)(3.14))$.

$$
\left|<\omega^{\sigma} v_{-}^{\prime}, \omega^{\sigma} L_{2} v_{-}^{\prime}>\right| \leq N(t)\left\|\omega^{\sigma} v_{-}^{\prime}\right\|^{2}
$$

We next estimate the second scalar product in (5.4) and we estimate the contribution of the various terms of (5.3) successively. We first estimate the contribution of $s_{-} \cdot \nabla$, namely

$$
M=\left|<\omega^{\sigma} v_{-}^{\prime}, \omega^{\sigma} s_{-} \cdot \nabla v_{1}^{\prime}>\right| .
$$

We consider separately the cases $\sigma \geq \rho-1$ and $\sigma<\rho-1$. For $\sigma \geq \rho-1$, we estimate

$$
M \leq C\left\|\omega^{2 \sigma+1-\rho} v_{-}^{\prime}\right\|\left(\left\|s_{-}\right\|_{\infty}+\left\|\omega^{n / 2} s_{-}\right\|\right)\left\|\omega^{\rho} v_{1}^{\prime}\right\|
$$

by Lemma 2.2. We next try to estimate

$$
\begin{aligned}
\left\|s_{-}\right\|_{\infty}+\left\|\omega^{n / 2} s_{-}\right\| & \leq C t^{\gamma-1}\left\|\omega^{1+\gamma-n / 2 \pm \varepsilon} \chi_{L}\left|v_{0}\right|_{-}^{2}\right\| \\
& \leq C t^{\gamma-1}\left\|\omega^{\rho_{2 \pm}} v_{0+}\right\|\left\|\omega^{\sigma_{2}} v_{0-}\right\|
\end{aligned}
$$

by Lemma 2.3 , where $\left|v_{0}\right|_{-}^{2} \equiv\left(\left|v_{0}\right|^{2}\right)_{-}$, with

$$
0 \leq \rho_{2 \pm} \leq \rho, 0 \leq \sigma_{2} \leq \sigma, \rho_{2 \pm}+\sigma_{2}=1+\gamma \pm \varepsilon
$$


which implies $\sigma_{2} \leq 1+\gamma-\varepsilon$. We choose $\sigma_{2}=\sigma \wedge(1+\gamma-\varepsilon)$.

If $\sigma \geq 1+\gamma-\varepsilon$, then $\sigma_{2}=1+\gamma-\varepsilon$ and $\rho_{2 \pm}=\varepsilon \pm \varepsilon \leq \rho$.

If $\sigma \leq 1+\gamma-\varepsilon$, then $\sigma_{2}=\sigma$ and $\rho_{2 \pm}=1+\gamma-\sigma \pm \varepsilon$, which satisfies $\rho_{2 \pm} \leq \rho$ for $\rho+\sigma>1+\gamma$. For $\rho+\sigma<1+\gamma$, we use the cut off $\chi_{L}$ to replace $\rho_{2 \pm}$ by $\rho$, so that

$$
\begin{aligned}
\left\|s_{-}\right\|_{\infty}+\left\|\omega^{n / 2} s_{-}\right\| & \leq C t^{\gamma-1-(1 / 2)[1+\gamma-\rho-\sigma]_{+}}\left\|v_{0+} ; H^{\rho}\right\| \\
& \times\left\|\omega^{\sigma \wedge(1+\gamma-\varepsilon)} v_{0-}\right\| .
\end{aligned}
$$

Now $\sigma \geq \rho-1$ implies $1+\gamma-\rho-\sigma \leq 2+\gamma-2 \rho$. Substituting (5.10) into (5.7) then yields

$$
M \leq C t^{\lambda_{1}-1} a a^{\prime}\left\|\omega^{2 \sigma+1-\rho} v_{-}^{\prime}\right\|\left\|\omega^{\sigma \wedge(1+\gamma-\varepsilon)} v_{0-}\right\| .
$$

The first norm in (5.11) is controlled by the $H^{\rho}$ norm of $v_{-}^{\prime}$ only if $\sigma \leq \rho-1 / 2$, and that is the origin of that condition, which is otherwise not used to derive (5.11).

We next estimate $M$ in the case where $\sigma<\rho-1$ which we rewrite as $\sigma \leq \rho-1-\varepsilon$. We estimate

$$
M \leq C\left\|\omega^{\sigma} v_{-}^{\prime}\right\|\left\|\omega^{n / 2-\rho_{1}+1+\sigma} s_{-}\right\|\left\|\omega^{\rho_{1}} v_{1}^{\prime}\right\|
$$

by Lemma 2.3 for

$$
0 \leq \sigma \leq \rho_{1}-1-\varepsilon
$$

We next try to estimate

$$
\begin{aligned}
& \left\|\omega^{n / 2-\rho_{1}+1+\sigma} s_{-}\right\|=C t^{\gamma-1}\left\|\omega^{2+\gamma-\rho_{1}+\sigma-n / 2} \chi_{L}\left|v_{0}\right|_{-}^{2}\right\| \\
& \leq C t^{\gamma-1}\left\|\omega^{\rho_{2}} v_{0+}\right\|\left\|\omega^{\sigma_{2}} v_{0-}\right\|
\end{aligned}
$$

by Lemma 2.3 again, with

$$
0 \leq \rho_{1}, \rho_{2} \leq \rho, 0 \leq \sigma_{2} \leq \sigma, \rho_{2}+\sigma_{2}=2+\gamma-\rho_{1}+\sigma
$$

which together with (5.13), implies $\sigma_{2} \leq 1+\gamma-\varepsilon$. We choose again $\sigma_{2}=\sigma \wedge(1+\gamma-\varepsilon)$.

If $\sigma \geq 1+\gamma-\varepsilon$, then $\sigma_{2}=1+\gamma-\varepsilon$ and $\rho_{1}+\rho_{2}=1+\sigma+\varepsilon$. We choose $\rho_{2}=0$ and $\rho_{1}=1+\sigma+\varepsilon$, so that $\rho_{2}<\rho_{1} \leq \rho$.

If $\sigma \leq 1+\gamma-\varepsilon$, then $\sigma_{2}=\sigma$ and $\rho_{1}+\rho_{2}=2+\gamma$. We choose

$$
\left\{\begin{array}{l}
\rho_{1}=(1+\gamma / 2) \vee(1+\sigma+\varepsilon) \\
\rho_{2}=(1+\gamma / 2) \wedge(1+\gamma-\sigma-\varepsilon)
\end{array}\right.
$$


which implies $\rho_{2} \leq \rho_{1} \leq \rho$ and therefore ensures (5.14), except in the case where $\sigma<\gamma / 2-\varepsilon$ and $\rho<1+\gamma / 2$. In that case, we use the cut off $\chi_{L}$ to replace $\rho_{1}$ and $\rho_{2}$ by $\rho$, so that

$$
\left\|\omega^{n / 2-\rho+1+\sigma} s_{-}\right\| \leq C t^{\lambda_{1}-1}\left\|\omega^{\rho} v_{0+}\right\|\left\|\omega^{\sigma} v_{0-}\right\|
$$

Substituting (5.14) or (5.17) into (5.12), together with (5.11), yields the estimate

$$
M \leq C t^{\lambda_{1}-1} a a^{\prime}\left\|\omega^{\sigma \vee(2 \sigma+1-\rho)} v_{-}^{\prime}\right\|\left\|\omega^{\sigma \wedge(1+\gamma-\varepsilon)} v_{0-}\right\|
$$

for $0 \leq \sigma<\rho$.

We next turn to the remaining terms from (5.3). They take the form

$$
<\omega^{\sigma} v_{-}^{\prime}, \omega^{\sigma} F v_{1}^{\prime}>
$$

for some function $F$ and are estimated as

$$
\left|<\omega^{\sigma} v_{-}^{\prime}, \omega^{\sigma} F v_{1}^{\prime}>\right| \leq C\left\|\omega^{\sigma} v_{-}^{\prime}\right\|\left\|\omega^{n / 2-\rho_{1}+\sigma} F\right\|\left\|\omega^{\rho_{1}} v_{1}^{\prime}\right\|
$$

by Lemma 2.3 , for some $\rho_{1}, 0 \leq \rho_{1} \leq \rho$, with

$$
0 \leq \sigma \leq \rho_{1}-\varepsilon
$$

We now estimate the middle norm in the RHS of (5.19) for the relevant choices of $F$ and suitable choices of $\rho_{1}$.

We first consider the contribution of $\nabla \cdot s_{-}$. We try to estimate

$$
\begin{aligned}
\left\|\omega^{n / 2-\rho_{1}+\sigma} \nabla \cdot s_{-}\right\| & \leq C t^{\gamma-1}\left\|\omega^{2+\gamma-\rho_{1}+\sigma-n / 2} \chi_{L}\left|v_{0}\right|_{-}^{2}\right\| \\
& \leq C t^{\gamma-1}\left\|\omega^{\rho_{2}} v_{0+}\right\|\left\|\omega^{\sigma_{2}} v_{0-}\right\|
\end{aligned}
$$

with $\rho_{1}, \rho_{2}, \sigma_{2}$ satisfying (5.20) (5.15), which imply $\sigma_{2} \leq 2+\gamma-\varepsilon$. We choose $\sigma_{2}=\sigma \wedge(2+\gamma-\varepsilon)$.

If $\sigma \geq 2+\gamma-\varepsilon$, we choose $\rho_{1}=\sigma+\varepsilon$ and $\rho_{2}=0$.

If $\sigma \leq 2+\gamma-\varepsilon$, so that $\sigma_{2}=\sigma$, we choose

$$
\left\{\begin{array}{l}
\rho_{1}=(1+\gamma / 2) \vee(\sigma+\varepsilon) \\
\rho_{2}=(1+\gamma / 2) \wedge(2+\gamma-\sigma-\varepsilon)
\end{array}\right.
$$

which ensures (5.20) (5.15) and (5.21) for $\rho \geq 1+\gamma / 2$. For $\rho<1+\gamma / 2$, we use the cut off $\chi_{L}$ to replace $\rho_{1}$ and $\rho_{2}$ by $\rho$, so that finally

$$
\left\|\omega^{n / 2-\rho_{1}+\sigma} \nabla \cdot s_{-}\right\| \leq C t^{\lambda_{1}-1} a\left\|\omega^{\sigma \wedge(2+\gamma-\varepsilon)} v_{0-}\right\| .
$$


We next consider the contribution of the various terms of $f_{-}$, where $f$ is defined by (3.6) . We first consider the contribution of $|s|_{-}^{2}\left(\equiv\left(|s|^{2}\right)_{-}\right)$. We estimate

$$
\left\|\omega^{n / 2-\rho_{1}+\sigma}|s|_{-}^{2}\right\| \leq C\left\|\omega^{n / 2 \pm 0} s_{+}\right\|\left\|\omega^{n / 2-\rho_{1}+\sigma} s_{-}\right\|
$$

by Lemma 2.2. The first norm in the RHS is estimated by (2.9). We try to estimate the second norm by

$$
\begin{aligned}
& \left\|\omega^{n / 2-\rho_{1}+\sigma} s_{-}\right\| \leq C t^{\gamma-1}\left\|\omega^{1+\gamma-\rho_{1}+\sigma-n / 2} \chi_{L}\left|v_{0}\right|_{-}^{2}\right\| \\
& \leq C t^{\gamma-1}\left\|\omega^{\rho_{2}} v_{0+}\right\|\left\|\omega^{\sigma_{2}} v_{0-}\right\|
\end{aligned}
$$

with $\rho_{1}, \rho_{2}, \sigma_{2}$ satisfying (5.20) and

$$
0 \leq \rho_{1}, \rho_{2} \leq \rho, 0 \leq \sigma_{2} \leq \sigma, \rho_{2}+\sigma_{2}=1+\gamma-\rho_{1}+\sigma
$$

which imply $\sigma_{2} \leq 1+\gamma-\varepsilon$. We choose $\sigma_{2}=\sigma \wedge(1+\gamma-\varepsilon)$.

If $\sigma \geq 1+\gamma-\varepsilon$, we choose $\rho_{1}=\sigma+\varepsilon$ and $\rho_{2}=0$.

If $\sigma \leq 1+\gamma-\varepsilon$, so that $\sigma_{2}=\sigma$, we choose

$$
\left\{\begin{array}{l}
\rho_{1}=(1+\gamma) / 2 \vee(\sigma+\varepsilon) \\
\rho_{2}=(1+\gamma) / 2 \wedge(1+\gamma-\sigma-\varepsilon)
\end{array}\right.
$$

which ensures (5.20) (5.26) and (5.25) for $\rho \geq(1+\gamma) / 2$. For $\rho<(1+\gamma) / 2$, we use the cut-off $\chi_{L}$ to replace $\rho_{1}$ and $\rho_{2}$ by $\rho$ so that finally

$$
\left\|\omega^{n / 2-\rho_{1}+\sigma} s_{-}\right\| \leq C t^{\lambda_{0}-1} a\left\|\omega^{\sigma \wedge(1+\gamma-\varepsilon)} v_{0-}\right\|
$$

and

$$
\left\|\omega^{n / 2-\rho_{1}+\sigma}|s|_{-}^{2}\right\| \leq C t^{2 \lambda_{0}-2} a^{3}\left\|\omega^{\sigma \wedge(1+\gamma-\varepsilon)} v_{0-}\right\|
$$

We next estimate the contribution of $g_{S-}$ to $f_{-}$by

$$
\begin{aligned}
& t^{\gamma-2}\left\|\omega^{n / 2-\rho+\sigma} g_{S-}\right\|=C t^{\gamma-2}\left\|\omega^{\gamma-\rho+\sigma-n / 2} \chi_{S}|v|_{-}^{2}\right\| \\
& \leq C t^{\gamma-2+\rho-\gamma / 2}\left\|\omega^{\rho} v_{+}\right\|\left\|\omega^{\sigma} v_{-}\right\| \\
& \leq C t^{\lambda_{1}-1} a\left\|\omega^{\sigma} v_{-}\right\|
\end{aligned}
$$

for $\rho_{1}=\rho \geq \gamma / 2$.

Similarly

$$
\left\|\omega^{n / 2-\rho+\sigma}\left(t^{\gamma-2} g_{L}\left(v_{0}\right)_{-}-\partial_{t} \varphi_{-}\right)\right\| \leq C t^{\lambda_{1}-1} a\left\|\omega^{\sigma} v_{0-}\right\| .
$$


We next consider the contribution of $\left(g_{L}(v)-g_{L}\left(v_{0}\right)\right)_{-}$to $f_{-}$. We want to estimate

$$
\begin{aligned}
J & =t^{\gamma-2}\left\|\omega^{n / 2-\rho_{1}+\sigma}\left(g_{L}(v)-g_{L}\left(v_{0}\right)\right)_{-}\right\| \\
& =t^{\gamma-2}\left\|\omega^{\gamma-\rho_{1}+\sigma-n / 2} \chi_{L}\left(|v|_{-}^{2}-\left|v_{0}\right|_{-}^{2}\right)\right\| .
\end{aligned}
$$

From the conservation law (2.16), we obtain

$$
|v|_{-}^{2}-\left|v_{0}\right|_{-}^{2}=V_{1-}+V_{2-}+V_{3-}
$$

where

$$
\begin{aligned}
& V_{1-}=-\int_{0}^{t} d t^{\prime} \operatorname{Im}\left(\bar{v}_{+} \Delta v_{-}+\bar{v}_{-} \Delta v_{+}\right)\left(t^{\prime}\right) \\
& V_{2-}=\nabla \cdot \int_{0}^{t} d t^{\prime}\left(s_{+}|v|_{-}^{2}\right)\left(t^{\prime}\right) \\
& V_{3-}=\nabla \cdot \int_{0}^{t} d t^{\prime}\left(s_{-}|v|_{+}^{2}\right)\left(t^{\prime}\right) .
\end{aligned}
$$

We first consider the contribution of $V_{1-}$. By the same estimates as in Lemma 2.6, we obtain

$$
\left\|\omega^{\rho_{2}+\sigma_{2}-2-n / 2} V_{1-}\right\| \leq C \int_{0}^{t} d t^{\prime}\left\|\omega^{\rho_{2}} v_{+}\right\|\left\|\omega^{\sigma_{2}} v_{-}\right\|\left(t^{\prime}\right)
$$

for $0 \leq \rho_{2}, \sigma_{2}<n / 2,1<\rho_{2}+\sigma_{2} \leq 2+n / 2$. We try to estimate

$$
t^{\gamma-2}\left\|\omega^{\gamma-\rho_{1}+\sigma-n / 2} \chi_{L} V_{1-}\right\| \leq C t^{\gamma-2} \int_{0}^{t} d t^{\prime}\left(\left\|\omega^{\rho_{2}} v_{+}\right\|\left\|\omega^{\sigma_{2}} v_{-}\right\|\right)\left(t^{\prime}\right)
$$

with $\rho_{1}, \rho_{2}, \sigma_{2}$ satisfying (5.20) (5.15) and $\rho_{2}+\sigma_{2}>1$. We proceed as for the estimate of the contribution of $\nabla \cdot s_{-}$(see (5.21) (5.23)) and we obtain finally

$$
t^{\gamma-2}\left\|\omega^{\gamma-\rho_{1}+\sigma-n / 2} \chi_{L} V_{1-}\right\| \leq C t^{\lambda_{1}-2} a \int_{0}^{t} d t^{\prime}\left\|\omega^{\sigma \wedge(2+\gamma-\varepsilon)} v_{-}\left(t^{\prime}\right)\right\| .
$$

The condition $\rho_{2}+\sigma_{2}>1$ is ensured provided $\rho+\sigma>1$.

We shall also need an estimate of $\left\|v_{-}^{\prime}\right\|$. In all terms but $V_{1-}$ this is done by taking $\sigma=0$ in the available estimate. Doing so for $V_{1-}$ would require the stronger condition $\rho>1$. We shall instead estimate the corresponding norm of $V_{1-}$ in terms of the norm $\left\|\omega^{\sigma_{2}} v_{-}\right\|$for the previous choice of $\sigma_{2}$, thereby obtaining

$$
t^{\gamma-2}\left\|\omega^{\gamma-\rho_{1}-n / 2} \chi_{L} V_{1-}\right\| \leq C t^{\lambda_{1}-2} a \int_{0}^{t} d t^{\prime}\left\|\omega^{\sigma \wedge(2+\gamma-\varepsilon)} v_{-}\left(t^{\prime}\right)\right\|
$$

by a similar computation. 
We next consider the contribution of $V_{2-}$. We try to estimate

$$
\begin{aligned}
& t^{\gamma-2}\left\|\omega^{\gamma-\rho_{1}+\sigma-n / 2} \chi_{L} V_{2-}\right\| \\
& \leq t^{\gamma-2} \int_{0}^{t} d t^{\prime}\left\|\omega^{1+\gamma-\rho_{1}+\sigma-n / 2} \chi_{L}(t)\left(s_{+}|v|_{-}^{2}\right)\left(t^{\prime}\right)\right\| \\
& \leq C t^{\gamma-2} \int_{0}^{t} d t^{\prime}\left(\left(\left\|s_{+}\right\|_{\infty}+\left\|\omega^{n / 2} s_{+}\right\|\right)\left\|\omega^{\rho_{2}} v_{+}\right\|\left\|\omega^{\sigma_{2}} v_{-}\right\|\right)\left(t^{\prime}\right)
\end{aligned}
$$

with $\rho_{1}, \rho_{2}, \sigma_{2}$ satisfying (5.20) (5.26). We estimate the norms of $s_{+}$by (2.9) and we proceed for the remaining norms as for the estimate of $s_{-}$(see (5.25) -(5.28) ), thereby obtaining finally

$$
\begin{aligned}
& t^{\gamma-2}\left\|\omega^{\gamma-\rho_{1}+\sigma-n / 2} \chi_{L} V_{2-}\right\| \\
& \leq C t^{\lambda_{0}-2} a^{3} \int_{0}^{t} d t^{\prime} t^{\prime \lambda_{0}-1}\left\|\omega^{\sigma \wedge(1+\gamma-\varepsilon)} v_{-}\left(t^{\prime}\right)\right\| .
\end{aligned}
$$

We next consider the contribution of $V_{3-}$. We estimate

$$
\begin{aligned}
& t^{\gamma-2}\left\|\omega^{\gamma-\rho_{1}+\sigma-n / 2} \chi_{L} V_{3-}\right\| \\
& \leq t^{\gamma-2} \int_{0}^{t} d t^{\prime}\left\|\omega^{1+\gamma-\rho_{1}+\sigma-n / 2} \chi_{L}(t)\left(s_{-}|v|_{+}^{2}\right)\left(t^{\prime}\right)\right\| .
\end{aligned}
$$

For $\rho \geq(1+\gamma) / 2$, we estimate the last norm by

$$
\begin{aligned}
\|\cdot\| & \leq C t^{\prime \lambda_{0}-1}\left\|\omega^{1+\gamma-n / 2}|v|_{+}^{2}\right\|\left\|\omega^{n / 2-\rho_{1}+\sigma} s_{-}\right\| \\
& \leq C t^{\prime \lambda_{0}-1}\left\|\omega^{(1+\gamma) / 2} v_{+}\right\|^{2}\left\|\omega^{\rho_{2}} v_{0+}\right\|\left\|\omega^{\sigma \wedge(1+\gamma-\varepsilon)} v_{0-}\right\|
\end{aligned}
$$

by Lemma 2.3 and by (5.25) with the choice (5.27) of $\rho_{1}, \rho_{2}$. For $\rho<(1+\gamma) / 2$, we take $\rho_{1}=\rho$ and we use the cut off $\chi_{L}$ twice to estimate

$$
\begin{aligned}
& t^{\gamma-2}\left\|\omega^{1+\gamma-\rho+\sigma-n / 2} \chi_{L}(t)\left(s_{-}|v|_{+}^{2}\right)\left(t^{\prime}\right)\right\| \\
& \leq C t^{\lambda_{0}-2}\left\|\omega^{\rho+\sigma-n / 2}\left(s_{-}|v|_{+}^{2}\right)\left(t^{\prime}\right)\right\| \\
& \leq C t^{\lambda_{0}-2}\left\|\omega^{2 \rho-n / 2}|v|_{+}^{2}\right\|\left\|\omega^{\sigma-\rho+n / 2} s_{-}\left(t^{\prime}\right)\right\| \\
& \leq C t^{\lambda_{0}-2} t^{\prime \lambda_{0}-1}\left\|\omega^{\rho} v_{+}\right\|^{2}\left\|\omega^{\rho+\sigma-n / 2}\left|v_{0}\right|_{-}^{2}\right\| \\
& \leq C t^{\lambda_{0}-2} t^{\prime \lambda_{0}-1}\left\|\omega^{\rho} v_{+}\right\|^{2}\left\|\omega^{\rho} v_{0+}\right\|\left\|\omega^{\sigma} v_{0-}\right\|
\end{aligned}
$$

by repeated use of Lemma 2.3. Substituting (5.44) or (5.45) into (5.43) and integrating over time yields

$$
t^{\gamma-2}\left\|\omega^{\gamma-\rho_{1}+\sigma-n / 2} \chi_{L} V_{3-}\right\| \leq C t^{2 \lambda_{0}-2} a^{3}\left\|\omega^{\sigma \wedge(1+\gamma-\varepsilon)} v_{0-}\right\| .
$$


We substitute (5.23) (5.29) (5.30) (5.31) (5.40) (5.42) (5.46) into (5.19) and substitute the result as well as (5.5) and (5.18) into (5.4), thereby obtaining

$$
\begin{aligned}
& \left|\partial_{t}\left\|\omega^{\sigma} v_{-}^{\prime}(t)\right\|^{2}\right| \leq N(t)\left\|\omega^{\sigma} v_{-}^{\prime}\right\|^{2} \\
& +C a a^{\prime} t^{\lambda_{1}-1}\left(\left\|\omega^{\sigma \vee(2 \sigma+1-\rho)} v_{-}^{\prime}\right\|\left\|\omega^{\sigma \wedge(1+\gamma)_{-}} v_{0-}\right\|\right. \\
& +\left\|\omega^{\sigma} v_{-}^{\prime}\right\|\left(\left\|\omega^{\sigma \wedge(2+\gamma)_{-}} v_{0-}\right\|+\left\|\omega^{\sigma} v_{-}\right\|+\left\|\omega^{\sigma} v_{-}\right\|\right. \\
& \left.\left.+t^{-1} \int_{0}^{t} d t^{\prime}\left\|\omega^{\sigma \wedge(2+\gamma)_{-}} v_{-}\left(t^{\prime}\right)\right\|\right)\right) \\
& +C a^{3} a^{\prime} t^{2 \lambda_{0}-2}\left\|\omega^{\sigma} v_{-}^{\prime}\right\|\left(\left\|\omega^{\sigma \wedge(1+\gamma)_{-}} v_{0-}\right\|\right. \\
& \left.+t^{-\lambda_{0}} \int_{0}^{t} d t^{\prime} t^{\prime \lambda_{0}-1}\left\|\omega^{\sigma \wedge(1+\gamma)_{-}} v_{-}\left(t^{\prime}\right)\right\|\right)
\end{aligned}
$$

where $(j+\gamma)_{-}=(j+\gamma-\varepsilon)$ for $j=1,2$. Together with the similar estimate for $\sigma=0$ (see however the discussion after (5.39)), with (3.14) and the fact that the second norm of $v_{-}^{\prime}$ in the RHS is bounded by $a^{\prime}$ for $\sigma \leq \rho-1 / 2$, this yields (5.1).

Remark 5.1. All the estimates leading to (5.47) hold for $0 \leq \sigma<\rho$ except for the estimate of $V_{1-}$ which requires $\rho+\sigma>1$. On the other hand the estimate (5.18) coming from the transport term $s_{-} \cdot \nabla$ can be used only for $\sigma \leq \rho-1 / 2$. Those two conditions force the restriction $\rho>3 / 4$.

We can now state the continuity properties of the map $v_{0} \rightarrow v$ defined in Proposition 4.1.

Proposition 5.1. Let $3 / 4<\rho<n / 2$. Let $R>0$ and let $T$ be defined by (4.12). Let $B_{0}(R / 2)$ be the ball of radius $R / 2$ in $H^{\rho}$.

(1) Let $1-\rho<\sigma<\rho$ and $\sigma \geq 0$. Then the map $v_{0} \rightarrow v$ defined by Proposition 4.1 is continuous from $H^{\sigma}$ to $L^{\infty}\left((0, T], H^{\sigma}\right)$ uniformly for $v_{0} \in B_{0}(R / 2)$. Furthermore, for $1-\rho<\sigma \leq \rho-1 / 2$ and for two solutions $v_{i}, i=1,2$ of the equation (1.21) with $v_{i}(0) \equiv v_{i 0} \in B_{0}(R / 2)$ as obtained in that proposition, the following estimate holds for all $t \in(0, T]$

$$
\left\|v_{-}(t) ; H^{\sigma}\right\|^{2} \leq\left(1+C t^{\lambda} \exp \left(C t^{\lambda}\right)\right)\left(y_{0}+C t^{\lambda}\left(y_{0}+y_{0}^{1 / 2}\right)\right)
$$

where $\lambda=\lambda_{1} \wedge\left(2 \lambda_{0}-1\right)$ and $y_{0}=\left\|v_{0-} ; H^{\sigma}\right\|^{2}$. 
For $\rho-1 / 2<\sigma<\rho$, a similar estimate can be obtained by interpolation between (5.48) with $\sigma=\rho-1 / 2$ and boundedness in $H^{\rho}$.

(2) The map $v_{0} \rightarrow v$ defined by Proposition 4.1 is (pointwise) continuous from $H^{\rho}$ to $L^{\infty}\left((0, T], H^{\rho}\right)$ for $v_{0} \in B_{0}(R / 2)$.

Proof. Part (1). Let $v_{i}, i=1,2$, be two solutions of the equation (1.21) as defined above. Then (5.1) with $y^{\prime}=y$ and $a^{\prime}=a$ yields

$$
\begin{aligned}
\left|\partial_{t} y\right| \leq & C t^{\lambda-1}\left(y+y^{1 / 2} y_{0}^{1 / 2}+y_{0}^{1 / 2}+y^{1 / 2} t^{-1} \int_{0}^{t} d t^{\prime} y^{1 / 2}\left(t^{\prime}\right)\right. \\
& \left.+y^{1 / 2} t^{-\lambda_{0}} \int_{0}^{t} d t^{\prime} t^{\lambda_{0}-1} y^{1 / 2}\left(t^{\prime}\right)\right) .
\end{aligned}
$$

(The constant $C$ depends on $a$ through a factor $\left(a^{2}+a^{4}\right)$ ). Using the inequalities

$$
\begin{aligned}
& t^{-1} \int_{0}^{t} d t^{\prime} y^{1 / 2}\left(t^{\prime}\right) \leq\left(t^{-1} \int_{0}^{t} d t^{\prime} y\left(t^{\prime}\right)\right)^{1 / 2} \\
& t^{-\lambda_{0}} \int_{0}^{t} d t^{\prime} t^{\prime \lambda_{0}-1} y^{1 / 2}\left(t^{\prime}\right) \leq\left(\left(2 \lambda_{0}-1\right)^{-1} t^{-1} \int_{0}^{t} d t^{\prime} y\left(t^{\prime}\right)\right)^{1 / 2}
\end{aligned}
$$

yields

$$
\left|\partial_{t} y\right| \leq C t^{\lambda-1}\left(\eta+y+\lambda^{-1} t^{-1} \int_{0}^{t} d t^{\prime} y\left(t^{\prime}\right)\right)
$$

where $\eta=y_{0}+y_{0}^{1 / 2}$. Integration of (5.50) over time yields

$$
y \leq y_{0}+C\left(t^{\lambda} \eta+z\right)
$$

where the new constant $C$ now depends on $\lambda$, we have used the fact that $0<\lambda<1$, and

$$
z=\int_{0}^{t} d t^{\prime} t^{\prime \lambda-1} y\left(t^{\prime}\right)
$$

so that

$$
\partial_{t} z \leq t^{\lambda-1} y_{0}+C\left(t^{2 \lambda-1} \eta+t^{\lambda-1} z\right) .
$$

Integrating (5.53) and substituting the result into (5.51) yields (5.48) from which Part (1) follows.

$\underline{\text { Part (2). }}$. The proof is identical with that of Part (2) of Proposition 5.1 in [4]. 
We finally prove the continuity of the map $v_{0} \rightarrow u_{c}$ that follows from Proposition 5.1 .

Proposition 5.2. Let $3 / 4<\rho<n / 2$. Let $R>0$ and let $T$ be defined by (4.12). Let $B_{0}(R / 2)$ be the ball of radius $R / 2$ in $H^{\rho}$. Then the map $v_{0} \rightarrow u_{c}$ defined by Proposition 4.2 is continuous from $H^{\rho}$ to $L^{\infty}\left(\left[t_{1}, T\right], H^{\rho}\right)$ for $v_{0} \in B_{0}(R / 2)$ and for any $t_{1}, 0<t_{1}<T$.

Proof. By Proposition 5.1, part (2) and (1.12), it suffices to prove that the multiplication by $\exp (-i \varphi)$ is strongly continuous from $v_{0} \in H^{\rho}$ as an operator in $L^{\infty}\left(\left[t_{1}, T\right], H^{\rho}\right)$. Let $v_{o i} \in H^{\rho}, i=1,2$ and let $\varphi_{i}$ be the associated phases defined by (1.18). For fixed $v \in L^{\infty}\left(\left[t_{1}, T\right], H^{\rho}\right)$ and for all $t \in\left[t_{1}, T\right]$, we estimate

$$
\begin{aligned}
& \left\|\left(\exp \left(-i \varphi_{2}\right)-\exp \left(-i \varphi_{1}\right)\right) v ; H^{\rho}\right\| \leq \\
& C\left(\|\delta \varphi\|_{\infty}+\left\|\omega^{n / 2} \delta \varphi\right\|\left(1+\left\|\omega^{n / 2} \delta \varphi\right\|\right)^{[\rho]}\right)\left\|\exp \left(-i \varphi_{1}\right) v ; H^{\rho}\right\|
\end{aligned}
$$

where $\delta \varphi=\varphi_{2}-\varphi_{1}$ and it suffices to prove that $\varphi$ is a continuous function of $v_{0}$ in $L^{\infty}\left(\left[t_{1}, T\right], L^{\infty} \cap \dot{H}^{n / 2}\right)$. This follows from the fact that $\varphi$ is quadratic in $v_{0}$ and from estimates similar to (4.17).

\section{Appendix A1}

In this appendix, we prove a slightly more general version of Lemma 2.4 where we drop the requirement that the estimating spaces are homogeneous under dilations. This extension would be useful to treat the main problem of this paper in spaces $H^{\rho}$ with $\rho \geq n / 2$.

We introduce the usual notation for the standard Paley-Littlewood decomposition. We use the notation $\widehat{f}$ for the Fourier transform of $f$. Let $\widehat{\psi}_{0} \in \mathcal{C}_{0}^{\infty}\left(I R^{n}, I R^{+}\right)$, $\widehat{\psi}_{0}(\xi)=1$ for $|\xi| \leq 1, \widehat{\psi}_{0}(\xi)=0$ for $|\xi| \geq 2$. We define $\widehat{\varphi}_{0}(\xi)=\widehat{\psi}_{0}(\xi)-\widehat{\psi}_{0}(2 \xi)$, $\widehat{\psi}_{j}(\xi)=\widehat{\psi}_{0}\left(2^{-j} \xi\right)$ and $\widehat{\varphi}_{j}(\xi)=\widehat{\varphi}_{0}\left(2^{-j} \xi\right)$ for all $j \in \mathrm{Z}$. For any positive integer $\nu$, we define

$$
\widetilde{\varphi}_{j}^{(\nu)}=\sum_{|j-k| \leq \nu} \varphi_{k} .
$$

The superscript $\nu$ will be omitted for $\nu=1$. For any $u \in \mathcal{S}^{\prime}$, we define $u_{j}=\varphi_{j} \star u$, 
$\widetilde{u}_{j}^{(\nu)}=\widetilde{\varphi}_{j}^{(\nu)} \star u$ and $S_{j}(u)=\psi_{j} \star u$. We shall repeatedly use the estimates

$$
\left\|\omega^{\lambda} \widetilde{u}_{j}^{(\nu)}\right\|_{r} \leq\left\|\omega^{\lambda} \widetilde{\varphi}_{j}^{(\nu+1)}\right\|_{1}\left\|\widetilde{u}_{j}^{(\nu)}\right\|_{r}=2^{\lambda j}\left\|\omega^{\lambda} \widetilde{\varphi}_{0}^{(\nu+1)}\right\|_{1}\left\|\widetilde{u}_{j}^{(\nu)}\right\|_{r}
$$

which holds for all $\lambda \in \mathbb{R}$ and $1 \leq r \leq \infty$, and

$$
\left\|\omega^{\lambda} S_{j}(u)\right\|_{r} \leq\left\|\omega^{\lambda} \psi_{j}\right\|_{1}\|u\|_{r}=2^{\lambda j}\left\|\omega^{\lambda} \psi_{0}\right\|_{1}\|u\|_{r}
$$

which holds for all $\lambda \geq 0$ and $1 \leq r \leq \infty$.

We shall use the following elementary lemma.

Lemma A1.1. Let

$$
M=\int d \xi d \eta \widehat{f}(\xi, \eta) \widehat{u}(\xi) \widehat{v}(\eta) \widehat{m}(\xi-\eta) .
$$

Then

$$
|M| \leq\|f\|_{1}\|u\|_{r_{1}}\|v\|_{r_{2}}\|m\|_{r_{0}}
$$

for $1 \leq r_{i} \leq \infty, \sum 1 / r_{i}=1$.

Proof. By the definition of the Fourier transform

$$
M=\int d x d y f(x, y) \int d z u(-x-z) v(-y+z) m(z)
$$

so that

$$
|M| \leq \int d x d y|f(x, y)| \operatorname{Sup}_{x, y}\left|\int d z u(-x-z) v(-y+z) m(z)\right|
$$

from which (A1.3) follows by the Hölder inequality.

We want to estimate $<P_{1} u,\left[\omega^{\lambda}, m\right] P_{2} v>$ which up to inessential complex conjugation reduces to

$$
\begin{aligned}
& M=\int d \xi d \eta\left(P_{1} \widehat{u}\right)(\xi)\left(P_{2} \widehat{v}\right)(\eta) \widehat{m}(\xi-\eta)\left(|\xi|^{\lambda}-|\eta|^{\lambda}\right) \\
= & \sum_{j, k, \ell} \int d \xi d \eta\left(P_{1} \widehat{u}_{j}\right)(\xi)\left(P_{2} \widehat{v}_{k}\right)(\eta) \widehat{m}_{\ell}(\xi-\eta)\left(|\xi|^{\lambda}-|\eta|^{\lambda}\right)
\end{aligned}
$$

by introducing the Paley-Littlewood decompositions of $u, v, m$.

The basic estimate is the following lemma. 
Lemma A1.2. Let $\lambda>0$ and let $P_{1}, P_{2}$ satisfy the assumptions of Lemma 2.4. Then $M$ can be decomposed as a sum

$$
M=M_{1}+M_{2}+M_{3}+M_{4}
$$

where the $M_{i}$ 's satisfy the following estimates

$$
\begin{gathered}
\left|M_{1}\right|+\left|M_{3}\right| \leq C\left\|u ; \dot{B}_{r_{1}, 2}^{\theta \mu}\right\|\|v\|_{r_{2}}\left\|m ; \dot{B}_{r_{0}, 2}^{(1-\theta) \mu}\right\| \\
\left|M_{2}\right|+\left|M_{3}\right| \leq C\|u\|_{r_{1}}\left\|v ; \dot{B}_{r_{2}, 2}^{\theta \mu}\right\|\left\|m ; \dot{B}_{r_{0}, 2}^{(1-\theta) \mu}\right\| \\
\left|M_{4}\right| \leq C\left\|u ; \dot{B}_{r_{1}, 2}^{(\mu-\nu)}\right\|\left\|v ; \dot{B}_{r_{2}, 2}^{(1-\theta)(\mu-\nu)}\right\|\left\|\omega^{\nu-1} \nabla m\right\|_{r_{0}}
\end{gathered}
$$

where $\mu=\lambda+\alpha_{1}+\alpha_{2}, 0 \leq \nu \leq 1,1 \leq r_{i} \leq \infty, \sum 1 / r_{i}=1$ and $\theta \in \mathbb{R}$. The parameters $r_{i}$ and $\theta$ can be chosen independently in the estimates (A1.6)-(A1.8).

Proof. The decomposition (A1.5) is obtained by splitting the sum in (A1.4) into four regions.

Region 1 . That region is defined by the condition $k \leq j-3$, so that

$$
2^{j-2} \leq|\xi|-|\eta| \leq|\xi-\eta| \leq|\xi|+|\eta| \leq 2^{j+2}
$$

and therefore $|j-\ell| \leq 2$. We obtain

$$
\left.M_{1}=\sum_{j} \int d \xi d \eta\left(P_{1} \widehat{u}_{j}\right)(\xi)\left(S_{j-3} \widehat{\left(P_{2}\right.} v\right)\right)(\eta) \widehat{\widetilde{m}_{j}^{(2)}}(\xi-\eta)\left(|\xi|^{\lambda}-|\eta|^{\lambda}\right) .
$$

The contribution of $|\xi|^{\lambda}$ is estimated by (A1.1) (A1.2) and the homogeneity of $P_{1}$, $P_{2}$ as

$$
\begin{gathered}
\left|M_{1 \xi}\right| \leq \sum_{j}\left\|\omega^{\lambda} P_{1} u_{j}\right\|_{r_{1}}\left\|S_{j-3}\left(P_{2} v\right)\right\|_{r_{2}}\left\|\widetilde{m}_{j}^{(2)}\right\|_{r_{0}} \\
\leq\left\|\omega^{\lambda} P_{1} \widetilde{\varphi}_{0}\right\|_{1}\left\|P_{2} \psi_{0}\right\|_{1} \sum_{j} 2^{\mu j-3 \alpha_{2}}\left\|u_{j}\right\|_{r_{1}}\|v\|_{r_{2}}\left\|\widetilde{m}_{j}^{(2)}\right\|_{r_{0}} \\
\leq C\left\|u ; \dot{B}_{r_{1}, 2}^{\theta \mu}\right\|\|v\|_{r_{2}}\left\|m ; \dot{B}_{r_{0}, 2}^{(1-\theta) \mu}\right\| .
\end{gathered}
$$

The contribution of $|\eta|^{\lambda}$ is estimated as

$$
\begin{gathered}
\left|M_{1 \eta}\right| \leq \sum_{j}\left\|P_{1} u_{j}\right\|_{r_{1}}\left\|\omega^{\lambda} S_{j-3}\left(P_{2} v\right)\right\|_{r_{2}}\left\|\widetilde{m}_{j}^{(2)}\right\|_{r_{0}} \\
\leq\left\|P_{1} \widetilde{\varphi}_{0}\right\|_{1}\left\|\omega^{\lambda} P_{2} \psi_{0}\right\|_{1} \sum_{j} 2^{\mu(j-3)+3 \alpha_{1}}\left\|u_{j}\right\|_{r_{1}}\|v\|_{r_{2}}\left\|\widetilde{m}_{j}^{(2)}\right\|_{r_{0}}
\end{gathered}
$$


and is therefore estimated by the last member of (A1.10). This proves the estimate (A1.6) for $M_{1}$.

Region 2. That region is defined by the condition $j \leq k-3$. The estimate of $M_{2}$ is then obtained from that of $M_{1}$ by exchanging $P_{1}$ with $P_{2}$ and $u$ with $v$. This proves the estimate (A1.7) for $M_{2}$.

The remaining region $|j-k| \leq 2$ is split again into two regions 3 and 4 . The important term is $M_{4}$ for which the commutator produces a cancellation, and the estimate of $M_{4}$ requires that $\tau \xi+(1-\tau) \eta$ stays away from zero for $0 \leq \tau \leq 1$. The harmless term $M_{3}$ takes care of the situation where that condition is not satisfied.

Region 3. That region is defined by the conditions $|j-k| \leq 2$ and $\ell \geq j-4$. The first condition implies that $\ell \leq j+4$ so that $|\ell-j| \leq 4$ and therefore

$$
M_{3}=\sum_{j} \int d \xi d \eta\left(P_{1} \widehat{u}_{j}\right)(\xi)\left(\widehat{P_{2} \widetilde{v}_{j}^{(2)}}\right)(\eta) \widehat{\widetilde{m}_{j}^{(4)}}(\xi-\eta)\left(|\xi|^{\lambda}-|\eta|^{\lambda}\right)
$$

We estimate $M_{3}$ by

$$
\begin{gathered}
\left|M_{3}\right| \leq\left(\left\|\omega^{\lambda} P_{1} \widetilde{\varphi}_{0}\right\|_{1}\left\|P_{2} \widetilde{\varphi}_{0}^{(3)}\right\|_{1}+\left\|P_{1} \widetilde{\varphi}_{0}\right\|_{1}\left\|\omega^{\lambda} P_{2} \widetilde{\varphi}_{0}^{(3)}\right\|_{1}\right) \\
\times \sum_{j} 2^{\mu j}\left\|u_{j}\right\|_{r_{1}}\left\|\widetilde{v}_{j}^{(2)}\right\|_{r_{2}}\left\|\widetilde{m}_{j}^{(4)}\right\|_{r_{0}} \\
\leq C\left(\left(\left\|u ; \dot{B}_{r_{1}, 2}^{\theta \mu}\right\|\left\|v ; \dot{B}_{r_{2}, \infty}^{0}\right\|\right) \wedge\left(\left\|u ; \dot{B}_{r_{1}, \infty}^{0}\right\|\left\|v ; \dot{B}_{r_{2}, 2}^{\theta \mu}\right\|\right)\right)\left\|m ; \dot{B}_{r_{0}, 2}^{(1-\theta) \mu}\right\|
\end{gathered}
$$

from which the estimates (A1.6) and (A1.7) for $M_{3}$ follow.

Region 4. That region is defined by the conditions $|j-k| \leq 2$ and $\ell \leq j-5$, so that

$$
M_{4}=\sum_{j} \int d \xi d \eta\left(P_{1} \widehat{u}_{j}\right)(\xi)\left(\widehat{P_{2} \widetilde{v}_{j}^{(2)}}\right)(\eta)\left(\widehat{S_{j-5}(m)}\right)(\xi-\eta)\left(|\xi|^{\lambda}-|\eta|^{\lambda}\right) .
$$

We rewrite

$$
|\xi|^{\lambda}-|\eta|^{\lambda}=\lambda \int_{0}^{1} d \tau(\xi-\eta) \cdot(\tau \xi+(1-\tau) \eta)|\tau \xi+(1-\tau) \eta|^{\lambda-2}
$$

so that

$$
M_{4}=\sum_{j} \int d \xi d \eta \widehat{f}_{j}(\xi, \eta)\left(P_{1} \widehat{u}_{j}\right)(\xi)\left(\widehat{P_{2} \widetilde{v}_{j}^{(2)}}\right)(\eta)\left(\nabla \widehat{S_{j-5}}(m)\right)(\xi-\eta)
$$


where

$$
\widehat{f}_{j}(\xi, \eta)=-i \lambda \int_{0}^{1} d \tau \widehat{\varphi_{j}(\xi)} \widehat{\widetilde{\varphi}_{j}^{(3)}}(\eta) \widehat{\psi}_{j-4}(\xi-\eta)(\tau \xi+(1-\tau) \eta)|\tau \xi+(1-\tau) \eta|^{\lambda-2}
$$

On the support of $\widehat{f}_{j}$, we have

$$
|\xi-\eta| \leq 2^{j-3} \leq|\xi| / 2
$$

so that

$$
|\tau \xi+(1-\tau) \eta| \geq|\xi|-|\xi-\eta| \geq|\xi| / 2
$$

and therefore $\widehat{f}_{j} \in \mathcal{C}_{0}^{\infty}$. We then estimate by Lemma A1.1 and the fact that $\widehat{f}_{j}$ is homogeneous of degree $\lambda-1$

$$
\left|M_{4}\right| \leq \sum_{j}\left\|f_{j}\right\|_{1}\left\|P_{1} u_{j}\right\|_{r_{1}}\left\|P_{2} \widetilde{v}_{j}^{(2)}\right\|_{r_{2}}\left\|S_{j-5}(\nabla m)\right\|_{r_{0}}
$$

$\leq\left\|f_{0}\right\|_{1}\left\|P_{1} \widetilde{\varphi}_{0}\right\|_{1}\left\|P_{2} \widetilde{\varphi}_{0}^{(3)}\right\|_{1}\left\|\omega^{1-\nu} \psi_{0}\right\|_{1} \sum_{j} 2^{(\mu-\nu) j}\left\|u_{j}\right\|_{r_{1}}\left\|\widetilde{v}_{j}^{(2)}\right\|_{r_{2}}\left\|\omega^{\nu-1} \nabla m\right\|_{r_{0}}$

from which the estimate (A1.8) follows.

Remark A1.1. The only assumptions needed on $P_{1}, P_{2}$ beyond homogeneity are the fact that $P_{i} \varphi_{0}, \omega^{\lambda} P_{i} \varphi_{0}, P_{i} \psi_{0}$ and $\omega^{\lambda} P_{i} \psi_{0}$ all belong to $L^{1}$. If the $P_{i}$ 's are smooth outside of the origin, this is obvious for $\varphi_{0}$, and follows from the dyadic decomposition of $\psi_{0}$ if $\alpha_{i}>0$. The assumptions made in Lemma 2.4 are trivially sufficient.

We now derive a slightly more general lemma than Lemma 2.4.

Lemma A1.3. Let $\lambda>0$ and let $P_{i}, i=1,2$, satisfy the assumptions of Lemma 2.4. Then the estimate (2.5) holds with

$$
\left\{\begin{array}{l}
\delta\left(q_{1}\right)=\mu+n / 2-\left(\sigma_{0}+\delta\left(r_{0}\right)+\sigma_{2}+\delta\left(r_{2}\right)\right) \\
\delta\left(q_{2}\right)=\mu+n / 2-\left(\sigma_{0}+\delta\left(r_{0}\right)+\sigma_{1}+\delta\left(r_{1}\right)\right) \\
\delta\left(q_{0}\right)=\mu-\nu+n / 2-\left(\sigma_{1}+\delta\left(r_{1}\right)+\sigma_{2}+\delta\left(r_{2}\right)\right)
\end{array}\right.
$$

under the conditions $0 \leq \nu \leq 1,1 \leq r_{i}, q_{i} \leq \infty$ and

$$
\left\{\begin{array}{l}
\sigma_{0}+\left(\sigma_{1} \wedge \sigma_{2}\right) \geq \mu \\
\sigma_{1}+\sigma_{2} \geq \mu-\nu
\end{array}\right.
$$


where $\mu=\lambda+\alpha_{1}+\alpha_{2}$ and $\delta(r)=n / 2-n / r$ for $1 \leq r \leq \infty$.

Proof. We rewrite the basic estimates (A1.6)-(A1.8) with a slightly different notation, namely

$$
\begin{aligned}
& \left|M_{1}\right|+\left|M_{3}\right| \leq C\left\|u ; \dot{B}_{s_{1}, 2}^{\mu_{1}}\right\|\|v\|_{q_{2}}\left\|m ; \dot{B}_{s_{0}, 2}^{\mu_{0}}\right\| \\
& \left|M_{2}\right|+\left|M_{3}\right| \leq C\|u\|_{q_{1}}\left\|v ; \dot{B}_{s_{2}, 2}^{\mu_{2}}\right\|\left\|m ; \dot{B}_{s_{0}^{\prime}, 2}^{\mu_{0}^{\prime}}\right\| \\
& \left|M_{4}\right| \leq C\left\|u ; \dot{B}_{s_{1}^{\prime}, 2}^{\mu_{1}^{\prime}}\right\|\left\|v ; \dot{B}_{s_{2}^{\prime}, 2}^{\mu_{2}^{\prime}}\right\|\left\|\omega^{\nu-1} \nabla m\right\|_{q_{0}}
\end{aligned}
$$

under the conditions $1 \leq s_{i}, s_{i}^{\prime}, q_{i} \leq \infty$,

$$
\left\{\begin{array}{l}
\mu_{1}+\mu_{0}=\mu_{2}+\mu_{0}^{\prime}=\mu \\
\mu_{1}^{\prime}+\mu_{2}^{\prime}=\mu-\nu
\end{array}\right.
$$

and the Hölder condition

$$
1 / s_{1}+1 / q_{2}+1 / s_{0}=1 / q_{1}+1 / s_{2}+1 / s_{0}^{\prime}=1 / s_{1}^{\prime}+1 / s_{2}^{\prime}+1 / q_{0}=1 \text {. }
$$

We want to choose the parameters $\mu_{i}, \mu_{i}^{\prime}, s_{i}, s_{i}^{\prime}$ in such a way that the $\dot{B}$ norms in (A1.19)-(A1.21) are controlled by the corresponding norms $\dot{B}$ in (2.5) through Sobolev inequalities. This holds provided

$$
\begin{gathered}
r_{i} \leq s_{i}, s_{i}^{\prime} \leq \infty \quad, \quad i=0,1,2, \\
\left\{\begin{array}{l}
\mu_{1}+\delta\left(s_{1}\right)=\mu_{1}^{\prime}+\delta\left(s_{1}^{\prime}\right)=\sigma_{1}+\delta\left(r_{1}\right) \\
\mu_{2}+\delta\left(s_{2}\right)=\mu_{2}^{\prime}+\delta\left(s_{2}^{\prime}\right)=\sigma_{2}+\delta\left(r_{2}\right) \\
\mu_{0}+\delta\left(s_{0}\right)=\mu_{0}^{\prime}+\delta\left(s_{0}^{\prime}\right)=\sigma_{0}+\delta\left(r_{0}\right) .
\end{array}\right.
\end{gathered}
$$

Eliminating $\mu_{i}, \mu_{i}^{\prime}$ between (A1.22) and (A1.25) yields

$$
\left\{\begin{array}{l}
\sigma_{0}+\sigma_{1}=\mu+\delta\left(s_{0}\right)-\delta\left(r_{0}\right)+\delta\left(s_{1}\right)-\delta\left(r_{1}\right) \\
\sigma_{0}+\sigma_{2}=\mu+\delta\left(s_{0}^{\prime}\right)-\delta\left(r_{0}\right)+\delta\left(s_{2}\right)-\delta\left(r_{2}\right) \\
\sigma_{1}+\sigma_{2}=\mu-\nu+\delta\left(s_{1}^{\prime}\right)-\delta\left(r_{1}\right)+\delta\left(s_{2}^{\prime}\right)-\delta\left(r_{2}\right) .
\end{array}\right.
$$


The conditions (A1.24) are then equivalent to

$$
\left\{\begin{array}{l}
\mu \leq \sigma_{0}+\sigma_{1} \leq \mu+n-\delta\left(r_{0}\right)-\delta\left(r_{1}\right) \\
\mu \leq \sigma_{0}+\sigma_{2} \leq \mu+n-\delta\left(r_{0}\right)-\delta\left(r_{2}\right) \\
\mu-\nu \leq \sigma_{1}+\sigma_{2} \leq \mu-\nu+n-\delta\left(r_{1}\right)-\delta\left(r_{2}\right) .
\end{array}\right.
$$

On the other hand, the Hölder condition (A1.23) can be rewritten as

$$
\delta\left(s_{1}\right)+\delta\left(q_{2}\right)+\delta\left(s_{0}\right)=\delta\left(q_{1}\right)+\delta\left(s_{2}\right)+\delta\left(s_{0}^{\prime}\right)=\delta\left(s_{1}^{\prime}\right)+\delta\left(s_{2}^{\prime}\right)+\delta\left(q_{0}\right)=n / 2
$$

and reduces to (A1.17) by the use of (A1.26). The left hand conditions of (A1.27) coincide with (A1.18) while the right hand conditions reduce to the already imposed conditions $q_{i} \geq 1$.

Lemma 2.4 is the special case of Lemma A1.3 where one imposes in addition the global homogeneity condition (2.7) under which (A1.17) reduces to (2.6).

\section{Appendix A2}

\section{Proof of Proposition 3.1.}

The proof proceeds by a parabolic regularization and a limiting procedure. We consider separately the cases $t \geq t_{0}$ and $t \leq t_{0}$ and we begin with $t \geq t_{0}$. We replace (1.23) by

$$
i \partial_{t} v^{\prime}=-(1 / 2)(1-i \eta) \Delta v^{\prime}+\widetilde{L} v^{\prime}
$$

where $\widetilde{L}$ is defined by $(\underline{3.16})$ and $0<\eta \leq 1$. We recast the Cauchy problem for (A2.1) with initial data $v^{\prime}\left(t_{0}\right)=v_{0}^{\prime}$ in the form of the integral equation

$$
v^{\prime}(t)=U_{\eta}\left(t-t_{0}\right) v_{0}^{\prime}-i \int_{t_{0}}^{t} d t^{\prime} U_{\eta}\left(t-t^{\prime}\right) \widetilde{L} v^{\prime}\left(t^{\prime}\right)
$$

where

$$
U_{\eta}(t)=\exp (i(t / 2)(1-i \eta) \Delta)
$$

We first solve (A2.2) locally in time by contraction in $\mathcal{C}\left(\left[t_{0}, t_{0}+T_{0}\right], H^{\rho^{\prime}}\right)$ for some $T_{0}>0$. The semi group $U_{\eta}$ satisfies the estimate

$$
\left\|U_{\eta}(t) \nabla v\right\| \leq(\eta t)^{-1 / 2}\|v\|
$$


so that by Lemmas 2.1 and 2.2

$$
\begin{aligned}
& \left\|\omega^{\sigma} U_{\eta}\left(t-t^{\prime}\right) \widetilde{L} v^{\prime}\left(t^{\prime}\right)\right\| \\
& \leq\left(\eta\left(t-t^{\prime}\right)\right)^{-1 / 2}\left\|\omega^{\sigma} s v^{\prime}\left(t^{\prime}\right)\right\|+\left\|\omega^{\sigma}(\nabla \cdot s) v^{\prime}\left(t^{\prime}\right)\right\|+\left\|\omega^{\sigma} f v^{\prime}\left(t^{\prime}\right)\right\| \\
& \leq C\left\{\left(\eta\left(t-t^{\prime}\right)\right)^{-1 / 2}\left\|\omega^{n / 2 \pm 0} s\right\|+\left\|\omega^{n / 2 \pm 0} \nabla s\right\|+\left\|\omega^{n / 2 \pm 0} f\right\|\right\}\left\|\omega^{\sigma} v^{\prime}\left(t^{\prime}\right)\right\|
\end{aligned}
$$

for $0 \leq \sigma \leq \rho^{\prime}$. We estimate the various terms in the same way as in the proof of Proposition 3.2 (see especially (3.8) (3.9)), except for the terms containing $g(v)$ or $g\left(v_{0}\right)$ for which we use the elementary estimate

$$
\left\|\omega^{n / 2 \pm 0} g\left(v_{(0)}\right)\right\| \leq C\left\|v_{(0)} ; H^{\rho}\right\|^{2}
$$

with $\rho>\gamma / 2$ instead of the more elaborate estimates (3.10)-(3.12). We can then continue (A2.4) as

$$
\begin{gathered}
\cdots \leq C\left\{\left(\eta\left(t-t^{\prime}\right)\right)^{-1 / 2} a_{0}^{2} t^{\prime \lambda_{0}-1}+a_{0}^{2}\left(t^{\prime \lambda_{1}-1}+a_{0}^{2} t^{\prime 2 \lambda_{0}-2}\right)\right. \\
\left.+\left(a_{0}^{2}+\left\|v\left(t^{\prime}\right) ; H^{\rho}\right\|^{2}\right) t^{\gamma-2}\right\}\left\|\omega^{\sigma} v^{\prime}\left(t^{\prime}\right)\right\|
\end{gathered}
$$

where $a_{0}=\left\|v_{0} ; H^{\rho}\right\|$. It then follows from (A2.6) that (A2.2) can be solved by contraction in $\mathcal{C}\left(\left[t_{0}, t_{0}+T_{0}\right], H^{\rho^{\prime}}\right)$ for $T_{0}$ sufficiently small. By a standard argument using the linearity of $(\mathrm{A} 2.2)$, one can extend the solution to $\left[t_{0}, T\right]$. Let $v_{\eta}^{\prime}$ be that solution.

We next take the limit where $\eta$ tends to zero. For that purpose we first estimate $v_{\eta}^{\prime}$ in $L^{\infty}\left(\left[t_{0}, T\right], H^{\rho^{\prime}}\right)$ uniformly in $\eta$. In the same way as in the proof of Proposition 3.2 , we estimate

$$
\begin{gathered}
\partial_{t}\left\|\omega^{\sigma} v_{\eta}^{\prime}(t)\right\|^{2}=-\eta\left\|\omega^{\sigma} \nabla v_{\eta}^{\prime}(t)\right\|^{2}+\operatorname{Im}<v_{\eta}^{\prime}(t),\left[\omega^{2 \sigma}, L(v)\right] v_{\eta}^{\prime}(t)> \\
\leq C\left(\left\|\omega^{n / 2} \nabla s\right\|+\|\nabla s\|_{\infty}+\left\|\omega^{n / 2} f\right\|\right)\left\|\omega^{\sigma} v_{\eta}^{\prime}(t)\right\|^{2} \\
\leq N_{0}(t)\left\|\omega^{\sigma} v_{\eta}^{\prime}(t)\right\|^{2}
\end{gathered}
$$

with

$$
N_{0}(t)=C\left\{a_{0}^{2}\left(t^{\lambda_{1}-1}+a_{0}^{2} t^{2 \lambda_{0}-2}\right)+\left(a_{0}^{2}+\left\|v(t) ; H^{\rho}\right\|^{2}\right) t^{\gamma-2}\right\}
$$

for $0 \leq \sigma \leq \rho^{\prime}$. Here we have again used (A2.5). It follows from (A2.7) by integration that $v_{\eta}^{\prime}$ is estimated in $L^{\infty}\left(\left[t_{0}, T\right], H^{\rho^{\prime}}\right)$ uniformly in $\eta$. By compactness, one can 
find a sequence of $v_{\eta}^{\prime}$ for $\eta$ tending to zero which converges in the weak- $\star$ sense to a limit $v^{\prime} \in L^{\infty}\left(\left[t_{0}, T\right], H^{\rho^{\prime}}\right)$. The limit $v^{\prime}$ satisfies the equation (1.23), so that $v^{\prime} \in \mathcal{C}\left(\left[t_{0}, T\right], H^{\rho^{\prime}-2}\right) \cap \mathcal{C}_{w}\left(\left[t_{0}, T\right], H^{\rho^{\prime}}\right)$. Furthermore $v_{\eta}^{\prime}$ tends to $v^{\prime}$ weakly in $H^{\rho^{\prime}}$ pointwise in $t$ so that $v^{\prime}\left(t_{0}\right)=v_{0}^{\prime}$. A similar argument yields the same results for $t \leq$ $t_{0}$. Uniqueness follows from $L^{2}$-norm conservation and linearity. Strong continuity in $H^{\rho^{\prime}}$ follows from the estimate (A2.7) which implies continuity of $\left\|v^{\prime}(t) ; H^{\rho^{\prime}}\right\|$ at $t_{0}$ and from uniqueness through a change of initial time.

\section{References}

[1] J. Bergh, J. Löfström, Interpolation Spaces, Springer, Berlin, 1976.

[2] J. Ginibre, G. Velo, Long range scattering and modified wave operators for some Hartree-type equations I, Rev. Math. Phys. 12 (2000) 361-429.

[3] J. Ginibre, G. Velo, Long range scattering and modified wave operators for some Hartree-type equations II, Ann. Henri Poincaré 1 (2000) 753-800.

[4] J. Ginibre, G. Velo, Long range scattering for the Wave-Schrödinger system revisited, J. Diff. Eq. 252 (2012) 1642-1667.

[5] K. Nakanishi, Modified wave operators for the Hartree equation with data, image and convergence in the same space, Commun. Pure Appl. Anal. 1 (2002) 237-252.

[6] K. Nakanishi, Modified wave operators for the Hartree equation with data, image and convergence in the same space II, Ann. Henri Poincaré 3 (2002) 503-535.

[7] E.M. Stein, Singular Integrals and Differentiability Properties of Functions, Princeton Univ. Press, Princeton, 1970. 